\title{
Decomposition in bunches of the critical locus of a quasi-ordinary map
}

\author{
E. R. García Barroso and P. D. González Pérez
}

\begin{abstract}
A polar hypersurface $P$ of a complex analytic hypersurface germ $f=0$ can be investigated by analyzing the invariance of certain Newton polyhedra associated with the image of $P$, with respect to suitable coordinates, by certain morphisms appropriately associated with $f$. We develop this general principle of Teissier when $f=0$ is a quasi-ordinary hypersurface germ and $P$ is the polar hypersurface associated with any quasi-ordinary projection of $f=0$. We show a decomposition of $P$ into bunches of branches which characterizes the embedded topological types of the irreducible components of $f=0$. This decomposition is also characterized by some properties of the strict transform of $P$ by the toric embedded resolution of $f=0$ given by the second author. In the plane curve case this result provides a simple algebraic proof of a theorem of Lê et al.
\end{abstract}

\section{Introduction}

The polar varieties or at least their rational equivalence classes play an important role in projective geometry, in particular in the study of characteristic classes and numerical invariants of projective algebraic varieties, and also in the study of projective duality (Plücker formulas). In the 1970s, local polar varieties began to be used systematically in the study of singularities. Local polar varieties can be used to produce invariants of equisingularity ('topological' invariants of complex analytic singularities) and also to explain why the same invariants appear in apparently unrelated questions. Here we study such equisingularity invariants and a particular instance of construction.

The Jacobian polygon, a plane polygon associated by Teissier to a germ of complex analytical hypersurface defining an isolated singularity at the origin, is an invariant of equisingularity, more precisely for the c-equisingularity. This property of a family of isolated hypersurface singularities is equivalent to Whitney conditions, implies topological triviality and is equivalent to it for plane curves. The inclinations of the compact edges of this polygon are rational numbers called the polar invariants of the germ. An isolated hypersurface singularity in $\mathbb{C}^{d+1}$ can be defined, with respect to suitable coordinates, by an equation $f\left(X_{1}, \ldots, X_{d+1}\right)=0$ in such a way that its Jacobian polygon coincides with the Newton polyhedron of image of the critical locus, or polar variety, of the morphism $\left(\mathbb{C}^{d+1}, 0\right) \rightarrow\left(\mathbb{C}^{2}, 0\right)$ defined by $T=f\left(X_{1}, \ldots, X_{d+1}\right)$ and $U=X_{1}$, with respect to the coordinates $(U, T)$ (see [Tei77b] and [Tei80]).

In the case of a germ of a plane irreducible curve, Merle showed that the polar invariants also determine the equisingularity class of the curve (or, equivalently, its embedded topological type), see [Mer77]. Merle's results were generalized to the case of reduced plane curve germs by Kuo and

Received 10 June 2003, accepted in final form 9 June 2004, published online 10 February 2005.

2000 Mathematics Subject Classification 14M25, 32S25.

Keywords: polar hypersurfaces, quasi-ordinary singularities, topological type, discriminants, toric geometry.

This research has been partly financed by 'Acción integrada hispano-francesa HF 2000-0119' and by 'Programme d'actions intégrées franco-espagnol 02685ND'. The second author was supported by a Marie Curie Fellowship of the European Community program 'Improving Human Research Potential and the Socio-economic Knowledge Base' under contract number HPMF-CT-2000-00877.

This journal is (C) Foundation Compositio Mathematica 2005. 


\section{E. R. García Barroso and P. D. González PÉrez}

Lu [KL77], Eggers [Egg82], García Barroso [Gar00] and Wall [Wal03] among others. They gave a decomposition theorem of a generic polar curve of a plane curve singularity, in bunches described by the equisingularity class of the curve. A matrix of partial polar invariants is associated with this decomposition, which determines the equisingularity class of the curve (see [Gar00]). Lê et al. have proved, using topological methods, that the strict transform of a generic polar curve by the minimal embedded resolution of the curve intersects the exceptional divisor inside a permitted Zariski open subset, and all connected components of the permitted subset are intersected (see [LMW89]). Another decomposition in bunches of the generic polar curve can be defined from this result in a geometrical way: each bunch corresponds with a connected component of the permitted subset. García Barroso has compared these two decompositions and shown that they coincide in [Gar00].

In this paper we study local polar hypersurfaces of a class of complex analytic hypersurface singularities, called quasi-ordinary. This class of singularities, of which the simplest example are the singularities of plane curves, appears naturally in Jung's approach to analyzing surface singularities and their parametrizations. A germ of complex analytic variety is quasi-ordinary if there exists a finite projection, called quasi-ordinary, to the complex affine space with discriminant locus contained in a normal crossing divisor. By Jung-Abhyankar's theorem any quasi-ordinary projection is provided with a parametrization with fractional power series [Jun08, Abh55]. In the hypersurface case these parametrizations possess a finite set of monomials, called characteristic or distinguished, which prescribe quite a lot of the geometry and topology of the singularity. For instance, these monomials constitute a complete invariant of its embedded topological type in the analytically irreducible case and conjecturally in the reduced case (see the works by Gau [Gau88] and Lipman [Lip83, Lip88]), in particular they determine the zeta function of the geometric monodromy as shown in the works of Némethi, McEwan and González Pérez (see [MN04, GMN03]). The characteristic monomials define embedded resolutions of the corresponding quasi-ordinary hypersurface singularity. This result has been obtained in two different ways by Villamayor [Vil00] and González Pérez [Gon02, Gon03].

We give a decomposition theorem of the polar hypersurface $(P, 0)$ of a quasi-ordinary hypersurface $(S, 0)$ corresponding to any quasi-ordinary projection. If $(S, 0)$ is embedded in $\left(\mathbb{C}^{d+1}, 0\right)$ any quasi-ordinary projection can be expressed in suitable coordinates by $\left(X_{1}, \ldots, X_{d}, Y\right) \mapsto$ $\left(X_{1}, \ldots, X_{d}\right)$. Then $(S, 0)$ has an equation defined by a Weierstrass polynomial $f \in \mathbb{C}\left\{X_{1}, \ldots, X_{d}\right\}$ $[Y]$ and the associated polar hypersurface $(P, 0)$ is defined as the critical space, $f_{Y}=0$, of the quasi-ordinary morphism:

$$
\left\{\begin{array}{l}
\xi_{f}:\left(\mathbb{C}^{d+1}, 0\right) \longrightarrow\left(\mathbb{C}^{d+1}, 0\right) \\
U_{1}=X_{1}, \ldots, U_{d}=X_{d}, \quad T=f\left(X_{1}, \ldots, X_{d}, Y\right) .
\end{array}\right.
$$

The decomposition is defined in terms of a matrix, generalizing the matrix of partial polar invariants of [Gar00], which determines and is determined by the partially ordered set of characteristic monomials associated with the fixed quasi-ordinary projection. In particular, it defines a complete invariant of the embedded topological type of each irreducible component of $(S, 0)$ by using Gau's and Lipman's results. Our decomposition theorem is partially motivated by a result of Popescu-Pampu providing a decomposition of the polar hypersurface $(P, 0)$ of the quasi-ordinary hypersurface $(S, 0)$, obtained with the additional hypothesis that $(S, 0)$ and $(P, 0)$ are simultaneously quasi-ordinary with respect to the given quasi-ordinary projection (see [Pop01, Chapter 3] or [Pop04]). Our decomposition extends also to the Laurent quasi-ordinary case, studied in [Pop01], by analogy to the case of meromorphic plane curves of Abhyankar and Assi (see [AA99]).

The proofs of the main results use an irreducibility criterion, for a power series with polygonal Newton polyhedron (the maximal dimension of its compact faces is equal to one), which generalizes a fundamental property of plane curve germs. Our criterion, which holds for power series with coefficients over any algebraically closed field of arbitrary characteristic, states that if an irreducible 
series has a polygonal Newton polyhedron, then it has only one compact edge. Our proof is obtained by using Newton polyhedra in the framework of toric geometry.

The decomposition theorem of the polar hypersurface $(P, 0)$ has a proof inspired by Teissier's works [Tei77b, Tei80]. In the irreducible case we analyze the discriminant $\mathcal{D}$ of the quasi-ordinary map $\xi_{f}$, i.e. the image of the critical space. We compute the Newton polyhedron $\mathcal{N}_{\mathcal{D}}$ of the discriminant $\mathcal{D}$ in the coordinates $U_{1}, \ldots, U_{d}, T$ above, and we show that it is a polygonal polyhedron. This computation applies the above-mentioned results of Popescu-Pampu, after suitable toric base changes already used in [Gon00a]. We then use the irreducibility criterion to show the existence of a decomposition of $(P, 0)$ in bunches that correspond bijectively to the compact edges of the polyhedron $\mathcal{N}_{\mathcal{D}}$.

We also give a geometrical characterization of the decomposition theorem by analyzing the strict transform of $(P, 0)$ by a modification, $p: \mathcal{Z} \rightarrow \mathbb{C}^{d+1}$, which is built canonically from the given quasiordinary projection by using the characteristic monomials (see [Gon03]). Geometrically, the bunches of the decomposition of $(P, 0)$ correspond to the union of branches of the polar hypersurface $(P, 0)$ whose strict transforms by $p$, meet the exceptional fiber $p^{-1}(0)$ at the same irreducible component. A posteriori this analysis can be extended to the toric embedded resolutions of $(S, 0)$ built in [Gon02] or [Gon03], since they are factored by $p$. In the plane curve case we apply this result to obtain a simple algebraic proof of the theorem of Lê et al. in [LMW89], which shows the underlying toric structure of the decomposition of the generic polar curve.

Our results provide answers to some of the questions raised independently by McEwan and Némethi in [MN03, $\S$ III], among some open problems concerning quasi-ordinary singularities. We hope that the results of this paper could apply to the study of polar varieties of hypersurface singularities by using a suitable form of Jung's approach. It is reasonable to expect that this work may have some applications to the metric study of the Milnor fibers of hypersurfaces, at least in the quasi-ordinary case, as suggested by García Barroso and Teissier's results in the case of plane curve singularities (see [GT99]); see also Risler's work [Ris03] for the real plane curve case.

The proofs are written in the analytic case. The results and proofs of this paper also hold in the algebroid case (over an algebraically closed field of characteristic zero).

\section{Quasi-ordinary polynomials, their characteristic monomials and the Eggers-Wall tree}

A germ of complex analytic hypersurface $(S, 0) \subset\left(\mathbb{C}^{d+1}, 0\right)$ is quasi-ordinary if there exists a finite projection $(S, 0) \rightarrow\left(\mathbb{C}^{d}, 0\right)$ that is a local isomorphism outside a normal crossing divisor. The embedding $(S, 0) \subset\left(\mathbb{C}^{d+1}, 0\right)$ can be defined by an equation $f=0$ where $f \in \mathbb{C}\{X\}[Y]$ is a quasiordinary polynomial: a Weierstrass polynomial with discriminant $\Delta_{Y} f$ of the form $\Delta_{Y} f=X^{\delta} \epsilon$ for a unit $\epsilon$ in the ring $\mathbb{C}\{X\}$ of convergent (or formal) power series in the variables $X=\left(X_{1}, \ldots, X_{d}\right)$ and $\delta \in \mathbb{Z}_{\geqslant 0}^{d}$.

The Jung-Abhyankar theorem guarantees that the roots of the quasi-ordinary polynomial $f$ are fractional power series in the ring $\mathbb{C}\left\{X^{1 / k}\right\}$ for some suitable integer $k$, for instance $k=\operatorname{deg} f$ when $f$ is irreducible (see $[\mathrm{Abh} 55]$ ). If the series $\left\{\zeta^{(l)}\right\}_{l=1}^{\operatorname{deg} f} \subset \mathbb{C}\left\{X^{1 / k}\right\}$ are the roots of $f$, the discriminant of $f$ with respect to $Y$ is equal to:

$$
\Delta_{Y} f=\prod_{i \neq j}\left(\zeta^{(i)}-\zeta^{(j)}\right),
$$

hence each factor $\zeta^{(t)}-\zeta^{(r)}$ is of the form $X^{\lambda_{t, r}} \epsilon_{t, r}$ where $\epsilon_{t, r}$ is a unit in $\mathbb{C}\left\{X^{1 / k}\right\}$. The monomials $X^{\lambda_{t, r}}$ (respectively the exponents $\lambda_{t, r}$ ) are called characteristic. 


\section{E. R. García Barroso and P. D. González Pérez}

In the reducible reduced case, if $f=f_{1} \ldots f_{s}$ is the factorization in monic irreducible polynomials each factor $f_{i}$ is a quasi-ordinary polynomial since $\Delta_{Y} f_{i}$ divides $\Delta_{Y} f$ by formula (1).

We define the partial order of $\mathbb{R}^{d} \cup\{+\infty\}$ :

$$
u \leqslant u^{\prime} \Leftrightarrow u^{\prime} \in u+\mathbb{R}_{\geqslant 0}^{d} .
$$

We write $u<u^{\prime}$ if $u \leqslant u^{\prime}$ and $u \neq u^{\prime}$. If $\alpha \in \mathbb{R}^{d}$ we set $\alpha<+\infty$. Note that $u \leqslant u^{\prime}$ means that the inequality holds coordinate-wise with respect to the canonical basis. The characteristic exponents have the following property with respect to the order (2), see [Lip88, Zar67].

LEMma 1. Let $f_{i}$ be an irreducible factor of the reduced quasi-ordinary polynomial $f$. The set

$$
V_{f}\left(f_{i}\right):=\left\{\lambda_{r, t} / \zeta^{(r)} \neq \zeta^{(t)}, f\left(\zeta^{(t)}\right)=0 \text { and } f_{i}\left(\zeta^{(r)}\right)=0\right\}
$$

is totally ordered by $\leqslant$.

If $f_{i}$ and $f_{j}$ are two irreducible factors of the quasi-ordinary polynomial $f$, using Lemma 1 we define the order of coincidence $k\left(f_{i}, f_{j}\right)$ of their roots by

$$
k\left(f_{i}, f_{j}\right)=\max \left\{\lambda_{r, t} / f_{i}\left(\zeta^{(r)}\right)=0, f_{j}\left(\zeta^{(t)}\right)=0\right\} .
$$

The order of coincidence of $f_{i}$ with itself is $k\left(f_{i}, f_{i}\right):=+\infty$. We have the following 'valuative' property of the orders of coincidence (see Lemma 3.10 of [Gon02]):

$$
\min \left\{k\left(f_{i}, f_{j}\right), k\left(f_{j}, f_{r}\right)\right\} \geqslant k\left(f_{i}, f_{r}\right) \quad \text { with equality if } k\left(f_{i}, f_{j}\right) \neq k\left(f_{j}, f_{r}\right) .
$$

The totally ordered set $V_{f}\left(f_{i}\right)$ defined by (3) is equal to the union of the non-necessarily disjoint sets whose elements are the characteristic exponents $\lambda_{1}^{(i)}<\cdots<\lambda_{g(i)}^{(i)}$ of $f_{i}$, if they exist, ${ }^{1}$ and the orders of coincidence $k\left(f_{i}, f_{j}\right)$ for $j=1, \ldots, s$ and $j \neq i$. We associate with the characteristic exponents of the irreducible factor $f_{i}$, for $i=1, \ldots, s$, the following sequences of characteristic lattices and integers: the lattices are $M_{0}^{(i)}:=\mathbb{Z}^{d}$ and $M_{j}^{(i)}:=M_{j-1}^{(i)}+\mathbb{Z} \lambda_{j}^{(i)}$ for $j=1, \ldots, g(i)$ with the convention $\lambda_{g(i)+1}^{(i)}=+\infty$; the integers are $n_{0}^{(i)}:=1$ and $n_{j}^{(i)}$ is the index of the subgroup $M_{j-1}^{(i)}$ in $M_{j}^{(i)}$, for $j=1, \ldots, g(i)$. We denote the integer $n_{j}^{(i)} \cdots n_{g(i)}^{(i)}$ by $e_{j-1}^{(i)}$ for $j=1, \ldots, g(i)$. We have that $\operatorname{deg} f_{i}=e_{0}^{(i)}=n_{1}^{(i)} \cdots n_{g(i)}^{(i)}\left(\operatorname{see}\left[\operatorname{Lip} 88\right.\right.$, Gon00b]). When $d=1$ we have the equality $M_{j}^{(i)}=\left(e_{j}^{(i)}\right) \mathbb{Z}$, and the integer $n_{j}^{(i)}$ coincides with the second component of the classical characteristic pairs of the plane branch defined by $f_{i}=0$. If $f$ is irreducible we drop the index $i$ in the notation above.

The information provided by the characteristic monomials is structured in a tree which encodes the embedded topological type of the irreducible components of $f=0$ (characterized by the works of Gau and Lipman in terms of the characteristic exponents, see [Gau88] and [Lip88]). In the quasi-ordinary case this tree was introduced by Popescu-Pampu (see [Pop01, Pop04]), following a construction of Wall [Wal03] and Eggers [Egg82] for plane curves.

The elementary branch $\theta_{f}\left(f_{i}\right)$ associated with $f_{i}$ is the abstract simplicial complex of dimension one with vertices running through the elements of the totally ordered subset $V_{f}\left(f_{i}\right) \cup\{0,+\infty\}$ of $\mathbb{Q}^{d} \cup\{\infty\}$, and edges running through the segments joining consecutive vertices. The underlying topological space is homeomorphic to the segment $[0,+\infty]$. We denote the vertex of $\theta_{f}\left(f_{i}\right)$ corresponding to $\lambda \in V_{f}\left(f_{i}\right) \cup\{0,+\infty\}$ by $P_{\lambda}^{(i)}$. The simplicial complex $\theta_{f}(f)$ obtained from the disjoint union $\bigsqcup_{i=1}^{s} \theta_{f}\left(f_{i}\right)$ by identifying in $\theta_{f}\left(f_{i}\right)$ and $\theta_{f}\left(f_{j}\right)$ the sub-simplicial complexes corresponding to

$$
\overline{P_{0}^{(i)} P_{k\left(f_{i}, f_{j}\right)}^{(i)}} \text { and } \overline{P_{0}^{(j)} P_{k\left(f_{i}, f_{j}\right)}^{(j)}}, \quad 1 \leqslant i<j \leqslant s,
$$

is a tree. We give the valuation $v\left(P_{\lambda}^{(i)}\right)=\lambda$ to a vertex $P_{\lambda}^{(i)}$ of $\theta_{f}(f)$.

\footnotetext{
${ }^{1}$ The case $f_{i}$ with no characteristic monomials happens only when $\operatorname{deg} f_{i}=1$.
} 


\section{DECOMPOSITION IN BUNCHES OF THE CRITICAL LOCUS OF A QUASI-ORDINARY MAP}

The set of vertices of $\theta_{f}(f)$ is partially ordered by $P \leqslant P^{\prime}$ if and only if $P, P^{\prime}$ are vertices of the same elementary branch of the tree and $v(P) \leqslant v\left(P^{\prime}\right)$. The valuation $v$ defines an orientation on the tree $\theta_{f}(f)$. The boundary operator $\partial$ is the linear map of integral 1-chains defined on the segments by $\partial\left(\overline{P P^{\prime}}\right)=P^{\prime}-P$ if $v(P)<v\left(P^{\prime}\right)$. For $i=1, \ldots, s$ we define an integral 1-chain whose segments are obtained by subdividing the segments of the chain

$$
\overline{P_{0}^{(i)} P_{\lambda_{1}^{(i)}}^{(i)}}+n_{1}^{(i)} \overline{P_{\lambda_{1}^{(i)}}^{(i)} P_{\lambda_{2}^{(i)}}^{(i)}}+\cdots+n_{1}^{(i)} \cdots n_{g(i)}^{(i)} \overline{P_{\lambda_{g(i)}^{(i)}}^{(i)} P_{+\infty}^{(i)}}
$$

with the points corresponding to the orders of coincidence of $f_{i}$, the coefficient of an oriented segment in the subdivision is the same as the coefficient of the oriented segment of (5) containing it. It follows that these 1-chains paste on $\theta_{f}(f)$ defining a 1-chain, which we denote by $\gamma_{f}$. The chain $\gamma_{f}$ characterizes the non-extremal vertices $P$ of $\theta_{f}\left(f_{i}\right)$ such that $v(P)$ is not a characteristic exponent of $f_{i}$. Any of these vertices $P$ appears in two segments of $\theta_{f}\left(f_{i}\right)$ with the same coefficient.

Definition 2. The Eggers-Wall tree of the quasi-ordinary polynomial $f$ is the simplicial complex $\theta_{f}(f)$ with the valuation $v$ and the chain $\gamma_{f}$.

Popescu-Pampu used the Eggers-Wall tree to represent the information provided by the orders of coincidence of the roots of $f$ with the roots of $h$ in the set $\mathcal{R} C_{f}$ of polynomials radically comparable with the polynomial $f$ (see [Pop04]):

$$
\mathcal{R} C_{f}:=\{h \in \mathbb{C}\{X\}[Y] / h \text { monic and the product } f h \text { is quasi-ordinary }\} .
$$

Any $h \in \mathcal{R} C_{f}$ is a quasi-ordinary polynomial and the difference of its roots with those of $f$ has a dominant monomial (viewed in $\mathbb{C}\left\{X^{1 / k}\right\}$ for some suitable $k$ ). If $h \in \mathcal{R} C_{f}$ we consider the sub-tree $\theta_{f h}(f)=\bigcup_{i=1}^{s} \theta_{f h}\left(f_{i}\right)$ of $\theta_{f h}(f h)$ as a subdivision of $\theta_{f}(f)$ induced by $h$. If $h$ is irreducible the point $P_{k\left(f_{i}, h\right)}^{(i)}$ is the point of bifurcation of the elementary branches $\theta_{f h}\left(f_{i}\right)$ and $\theta_{f h}(h)$ in $\theta_{f h}(f h)$; we denote the point of bifurcation of the elementary branch $\theta_{f h}(h)$ from the tree $\theta_{f h}(f)$ by $P_{k(h, f)}^{h}$. If $h \in \mathcal{R} C_{f}$ and if $h=h_{1} \ldots h_{t}$ is the factorization of $h$ as a product of monic irreducible polynomials, the contact chain $[h]^{(f)}$ is the integral 0 -chain on $\theta_{f h}(f)$ defined by

$$
[h]^{(f)}=\sum_{j=1}^{t} \operatorname{deg} h_{j} P_{k\left(h_{j}, f\right)}^{h_{j}} .
$$

The contact chain $[h]^{(f)}$ is associated with the decomposition $h=b_{1} \ldots b_{s(f, h)}$ in the ring $\mathbb{C}\{X\}[Y]$, where the factors $b_{j}$ are the products of those irreducible factors of $h$ having the same order of coincidence with each irreducible factor of $f$.

\section{Decomposition in bunches induced by a quasi-ordinary polynomial}

We show that a quasi-ordinary polynomial $f \in \mathbb{C}\{X\}[Y]$ defines in a natural way a decomposition in bunches for a certain class of polynomials that contains the derivative $f_{Y}$ of $f$. We characterize the decomposition in bunches of the polar hypersurface $f_{Y}=0$ and the Newton polyhedron of a power series defining the image of $f_{Y}=0$ by the quasi-ordinary morphism $\xi_{f}$, in terms of the tree $\theta_{f}(f)$.

If $f \in \mathbb{C}\{X\}[Y]$ is a quasi-ordinary polynomial the polynomial $f_{Y}:=n^{-1}(\partial f / \partial Y)$ belongs to the set $\mathcal{C}_{f}$ of polynomials comparable ${ }^{2}$ with $f$ :

$$
\mathcal{C}_{f}:=\left\{h \in \mathbb{C}\{X\}[Y] / h \text { monic, } \operatorname{Res}_{Y}(f, h)=X^{\rho(f, h)} \epsilon_{f, h} \text { with } \epsilon_{f, h}(0) \neq 0 \text { and } \rho(f, h) \in \mathbb{Z}^{d}\right\},
$$

where $\operatorname{Res}_{Y}(f, h)$ denotes the resultant of the polynomials $f$ and $h$.

${ }^{2}$ Our notion of comparable polynomials generalizes that of radically comparable (which corresponds to the notion of comparable polynomials of Popescu-Pampu in [Pop01, Pop04]). 


\section{E. R. García Barroso and P. D. González PÉrez}

We have an inclusion $\mathcal{R} C_{f} \subset \mathcal{C}_{f}$ : it is sufficient to note that $\operatorname{Res}_{Y}(f, h)$ divides $\Delta_{Y}(f h)$, a statement that follows from the classical properties of resultants and discriminants (see [GKZ94]):

$$
\operatorname{Res}_{Y}\left(f, h_{1} \cdots h_{t}\right)=\prod_{i=1}^{t} \operatorname{Res}_{Y}\left(f, h_{i}\right), \quad \Delta_{Y}(f h)=\Delta_{Y}(f) \Delta_{Y}(h)\left(\operatorname{Res}_{Y}(f, h)\right)^{2} .
$$

Remark 3. The inclusion $\mathcal{R} C_{f} \subset \mathcal{C}_{f}$ is strict in general. In particular, the derivative $f_{Y}$ of a quasiordinary polynomial $f \in \mathbb{C}\left\{X_{1}, \ldots, X_{d}\right\}[Y]$ is not radically comparable with $f$ in general.

This example has already been given by Popescu-Pampu. The polynomial $f=Y^{3}+X_{1} X_{2} Y^{2}+$ $X_{1}^{3} X_{2} Y+X_{1} X_{2}$ is quasi-ordinary thus $f_{Y} \in \mathcal{C}_{f}$ but $f_{Y} \notin \mathcal{R} C_{f}$ (see [Pop01, p. 127]).

If $f=f_{1} \cdots f_{s}$ is the factorization of $f$ in monic irreducible polynomials, we deduce from (6) and the definitions that $\mathcal{C}_{f}=\bigcap_{i=1}^{s} \mathcal{C}_{f_{i}}$. We define an equivalence relation in the set $\mathcal{C}_{f}$ : if $h, h^{\prime} \in \mathcal{C}_{f}$

$$
h \sim_{f} h^{\prime} \Leftrightarrow \frac{\rho\left(f_{i}, h\right)}{\operatorname{deg} h}=\frac{\rho\left(f_{i}, h^{\prime}\right)}{\operatorname{deg} h^{\prime}}, \quad \text { for } i=1, \ldots, s .
$$

By (6) if $h \in \mathcal{C}_{f}$ the irreducible factors of $h$ are also in $\mathcal{C}_{f}$. We denote by $s(f, h)$ the number of classes of the restriction of the equivalence relation (7) to the set of irreducible factors of $h$.

Definition 4. The $f$-bunch decomposition of a polynomial $h \in \mathbb{C}\left\{X_{1}, \ldots, X_{d}\right\}[Y]$ comparable with the quasi-ordinary polynomial $f \in \mathbb{C}\left\{X_{1}, \ldots, X_{d}\right\}[Y]$ is $h=b_{1} \cdots b_{s(f, h)}$, where the $b_{i}$, for $i=1, \ldots, s(f, h)$, are the products of the irreducible factors of $h$ that are in the same class. The type of the $f$-bunch decomposition of $h$ is the collection of vectors

$$
\left\{\left(\frac{\rho\left(f_{1}, h_{j}\right)}{\operatorname{deg} h_{j}}, \ldots, \frac{\rho\left(f_{s}, h_{j}\right)}{\operatorname{deg} h_{j}} ; \operatorname{deg} b_{j}\right)\right\}_{j=1}^{s(f, h)},
$$

where $h_{j}$ is any irreducible factor of $b_{j}$ for $j=1, \ldots, s(f, h)$.

If $f$ is clear from the context, we write bunch decomposition instead of $f$-bunch decomposition; in particular we do this for the polynomial $f_{Y}$. If $h \in \mathcal{R} C_{f}$ the type of the $f$-bunch decomposition of $h$ is studied by using the orders of coincidence of $h \in \mathcal{R} C_{f}$ with the irreducible factors of $f$.

We introduce some notation: if $\lambda \in V_{h f}\left(f_{i}\right)$ we denote by $q_{\lambda}^{(i)}$ the integer $q_{\lambda}^{(i)}=\max \left(\left\{j / \lambda_{j}^{(i)}<\lambda\right\}\right.$ $\cup\{0\})$, if $\lambda=k\left(h, f_{i}\right)$ we denote $q_{\lambda}^{(i)}$ by $q_{\left(h, f_{i}\right)}$. The following proposition extends the classical relation between the intersection multiplicity and the order of coincidence in the plane branch case (see Proposition 3.7.15 of [Pop01]).

Proposition 5. Let $h \in \mathcal{R} C_{f}$ be irreducible. If $\tau$ is any root of $h$ in $\mathbb{C}\left\{X^{1 / k}\right\}$ (for some integer $k>0)$ we have that $f_{i}(\tau)$ is of the form

$$
f_{i}(\tau)=X^{\rho\left(f_{i}, h\right) / \operatorname{deg} h} \epsilon_{i, \tau}
$$

where $\epsilon_{i, \tau}$ is a unit in $\mathbb{C}\left\{X^{1 / k}\right\}$ and

$$
\frac{\rho\left(f_{i}, h\right)}{\operatorname{deg} h}=e_{q_{\left(h, f_{i}\right)}^{(i)}}^{(i)}\left(h, f_{i}\right)+\sum_{k=1}^{q_{\left(h, f_{i}\right)}}\left(e_{k-1}^{(i)}-e_{k}^{(i)}\right) \lambda_{k}^{(i)} .
$$


Definition 6. We associate the valuation $\nu_{i}$ of the vertices of $\theta_{f h}(f)$ with the factor $f_{i}$ of $f$ :

$$
\nu_{i}\left(P_{\lambda}^{(j)}\right):= \begin{cases}e_{q_{\lambda}^{(i)}}^{(i)} \lambda+\sum_{k=1}^{q_{\lambda}^{(i)}}\left(e_{k-1}^{(i)}-e_{k}^{(i)}\right) \lambda_{k}^{(i)} & \text { if } P_{\lambda}^{(j)} \in \theta_{f h}\left(f_{i}\right), \lambda \neq 0,+\infty, \\ \nu_{i}\left(P_{k\left(f_{i}, f_{j}\right)}^{(i)}\right) & \text { if } P_{\lambda}^{(j)} \notin \theta_{f h}\left(f_{i}\right), \\ 0 & \text { if } \lambda=0, \\ +\infty & \text { if } j=i \text { and } \lambda=+\infty .\end{cases}
$$

Remark 7. If $h \in \mathcal{R} C_{f}$ is irreducible then $\nu_{i}\left(P_{k(h, f)}^{h}\right)=\rho\left(f_{i}, h\right) / \operatorname{deg} h$. If $f=f_{1}$ is irreducible we denote $\nu_{1}$ by $\nu$.

If $h \in \mathcal{C}_{f}$, in particular when $h=f_{Y}$, we study the $f$-bunch decomposition of $h$ by analyzing the Newton polyhedra, with respect to suitable coordinates, of the polynomials defining the images of $h=0$ under quasi-ordinary morphisms associated with the irreducible factors of $f$.

If $f \in \mathbb{C}\left\{X_{1}, \ldots, X_{d}\right\}[Y]$ is a quasi-ordinary polynomial we say that the morphism

$$
\left\{\begin{array}{l}
\xi_{f}:\left(\mathbb{C}^{d+1}, 0\right) \longrightarrow\left(\mathbb{C}^{d+1}, 0\right) \\
U_{1}=X_{1}, \ldots, U_{d}=X_{d}, \quad T=f\left(X_{1}, \ldots, X_{d}, Y\right)
\end{array}\right.
$$

is quasi-ordinary. By definition, the critical space of the morphism $\xi_{f}$ is the polar hypersurface, $f_{Y}=0$, associated with the given quasi-ordinary projection $\left(X_{1}, \ldots, X_{d}, Y\right) \mapsto\left(X_{1}, \ldots, X_{d}\right)$. The discriminant space is the image of the critical space by $\xi_{f}$, (see [Tei77a]).

More generally, if $h \in \mathbb{C}\{X\}[Y]$ the image of the hypersurface $h=0$ by $\xi_{f}$ is the hypersurface defined by $h=0, T-f=0, U_{1}=X_{1}, \ldots, U_{d}=X_{d}$. This image is defined by the vanishing of the series obtained by eliminating $X_{1}, \ldots, X_{d}, Y$, in the previous equations i.e., by

$$
\psi_{f}(h):=\operatorname{Res}_{Y}(T-f, h) .
$$

The degree of the polynomial $\psi_{f}(h) \in \mathbb{C}\{U\}[T]$ is equal to deg $h$. If $h=0$ is analytically irreducible at the origin the same holds for its image $\psi_{f}(h)=0$, thus $\psi_{f}(h)$ is an irreducible polynomial. If $h=h_{1} \cdots h_{t}$ then it follows from (6) that $\psi_{f}(h)=\prod_{r=1}^{t} \psi_{f}\left(h_{r}\right)$.

We analyze the Newton polyhedron of $\psi_{f_{i}}(h)$ for $f_{i}$ any irreducible factor of $f$. Recall that the Newton polyhedron $\mathcal{N}(\phi) \subset \mathbb{R}^{d}$ of a non zero series $\phi=\sum c_{\alpha} X^{\alpha} \in \mathbb{C}\{X\}$ with $X=\left(X_{1}, \ldots, X_{d}\right)$ is the convex hull of the set $\bigcup_{c_{\alpha} \neq 0} \alpha+\mathbb{R}_{\geqslant 0}^{d}$. The Newton polyhedron of a polynomial $F \in \mathbb{C}\{X\}[Y]$ is the polyhedron $\mathcal{N}(F) \subset \mathbb{R}^{d} \times \mathbb{R}$ of $F$ viewed as a series in $X_{1}, \ldots, X_{d}, Y$. We introduce the following notation:

Notation 8. We denote the Newton polyhedron of $Y^{p}-X^{a} \in \mathbb{C}\left\{X_{1}, \ldots, X_{d}\right\}[Y]$ by the symbol $\frac{p}{\bar{q}}$ (or, equivalently, $p / / q$ ) where $q:=a / p \in \mathbb{Q}^{d}$ is the inclination of the edge of $\mathcal{N}\left(Y^{p}-X^{a}\right)$. Our notation $\frac{p}{\bar{q}}$ is inspired by that used by Teissier $\left\{\frac{b}{\bar{a}}\right\}$ with a different meaning

$$
\left(\left\{\frac{b}{\bar{a}}\right\}:=\frac{b}{\overline{a / b}}\right)
$$

to describe elementary Newton polygons in [Tei77a, Tei80]. We have the following property of the Minkowski sum:

$$
\frac{p}{\bar{q}}+\frac{p^{\prime}}{\bar{q}}=\frac{p+p^{\prime}}{q} .
$$

We prove that the tree $\theta_{f}(f)$ determines the Newton polyhedra $\mathcal{N}\left(\psi_{f_{i}}\left(f_{Y}\right)\right)$ and $\mathcal{N}\left(\psi_{f}\left(f_{Y}\right)\right)$. 


\section{E. R. García Barroso and P. D. González PÉrez}

Theorem 1. Let $f \in \mathbb{C}\left\{X_{1}, \ldots, X_{d}\right\}[Y]$ be a quasi-ordinary polynomial, and $f=f_{1} \cdots f_{s}$ be the factorization of $f$ in monic irreducible polynomials. The Newton polyhedron of $\psi_{f_{i}}\left(f_{Y}\right)$ (respectively of $\left.\psi_{f}\left(f_{Y}\right)\right)$ is the Minkowski sum

$$
\mathcal{N}\left(\psi_{f_{i}}\left(f_{Y}\right)\right)=\sum_{j} \frac{c_{j}}{\overline{\nu_{i}\left(P_{j}\right)}} \quad\left(\text { respectively } \mathcal{N}\left(\psi_{f}\left(f_{Y}\right)\right)=\sum_{j} \frac{c_{j}}{\overline{\nu_{1}\left(P_{j}\right)+\cdots+\nu_{s}\left(P_{j}\right)}}\right),
$$

where in both cases $P_{j}$ runs through the set of non-extremal vertices of $\theta_{f}(f)$ and $c_{j}$ is the coefficient of $P_{j}$ in the chain $-\partial \gamma_{f}$.

We use this result to characterize the type of the bunch decomposition of the polar hypersurface $f_{Y}=0$ in terms of the tree $\theta_{f}(f)$. Conversely, we show that the type of the decomposition of $f_{Y}=0$ and the degrees of the irreducible factors of $f$ determines the Eggers-Wall tree (this result generalizes a theorem of García Barroso for a generic polar curve, see Théorème 6.1 of [Gar00]).

Theorem 2. Let $f \in \mathbb{C}\left\{X_{1}, \ldots, X_{d}\right\}[Y]$ be a quasi-ordinary polynomial with monic irreducible factors $f_{1}, \ldots, f_{s}$.

(i) The type of the bunch decomposition of the partial derivative $f_{Y}$ is

$$
\left\{\left(\nu_{1}\left(P_{j}\right), \ldots, \nu_{s}\left(P_{j}\right) ; c_{j}\right)\right\}_{j}
$$

where $P_{j}$ runs through the set of non-extremal vertices of $\theta_{f}(f)$ and $c_{j}$ is the coefficient of $P_{j}$ in the chain $-\partial \gamma_{f}$. In particular, when $f$ is irreducible with characteristic exponents $\lambda_{1}, \ldots, \lambda_{g}$, the type of $f_{Y}$ is

$$
\left\{\left(\nu\left(P_{\lambda_{j}}\right) ; n_{0} n_{1} \cdots n_{j-1}\left(n_{j}-1\right)\right)\right\}_{j=1}^{g} .
$$

(ii) The type of the bunch decomposition of $f_{Y}$ and the degrees of the irreducible factors of $f$ determine the Eggers-Wall tree of $f$.

Remark 9. Assertion (i) of Theorem 2 generalizes Popescu-Pampu's Theorem 3.8.5 of [Pop01] (or Theorem 6.3 of [Pop04]) obtained in the case of a quasi-ordinary derivative (when $f_{Y} \in \mathcal{R} C_{f}$ ).

In Popescu-Pampu's paper [Pop04] the approach of Wall [Wal03] is extended to the quasiordinary case. Essential to this extension is a generalization of a lemma of Kuo and Lu [KL77, Lemma 3.3], which is also essential in [Egg82, Gar00, Wal03]. Kuo-Lu's lemma, in the case of a plane curve germ defined by a Weierstrass polynomial $F \in \mathbb{C}\{X\}[Y]$, compares the dominant terms of the differences of any fixed root $Y=\zeta(X)$ of $F$, with the other roots of $F$, and the differences of $\zeta(X)$ with the roots of $F_{Y}$. The additional hypothesis needed to generalize Kuo-Lu's lemma to the case of a quasi-ordinary polynomial $f \in \mathbb{C}\left\{X_{1}, \ldots, X_{d}\right\}[Y]$, i.e. to compare the roots of the derivative $f_{Y}$ with the roots of $f$, is that the polynomial $f_{Y}$ should be radically comparable with $f$. If $f_{Y} \notin \mathcal{R} C_{f}$, the quasi-ordinary projection $\left(X_{1}, \ldots, X_{d}, Y\right) \mapsto\left(X_{1}, \ldots, X_{d}\right)$ may be replaced by a base change defined by an embedded resolution of the discriminant $\Delta_{Y}\left(f_{Y} \cdot f\right)=0$, in such a way that the transforms of $f$ and $f_{Y}$ become simultaneously quasi-ordinary with respect to the same quasi-ordinary projection over any point of the exceptional divisor. However, it is not clear that the decompositions obtained in this way come from a decomposition of $f_{Y}=0$, since base changes do not preserve irreducible components in general.

\section{Newton polyhedra and toric geometry}

In the following sections we introduce the tools needed to prove the main results. 


\section{DECOMPOSITION IN BUNCHES OF THE CRITICAL LOCUS OF A QUASI-ORDINARY MAP}

\subsection{Polygonal Newton polyhedra and their dual Newton diagrams}

If $\phi \in \mathbb{C}\{X\}$ is a non-zero series in the variables $X=\left(X_{1}, \ldots, X_{d}\right)$ we have that any linear form $w \in\left(\mathbb{R}^{d}\right)^{*}$ in the cone $\Delta_{d}:=\left(\mathbb{R}^{d}\right)_{\geqslant 0}^{*}$ defines a face $\mathcal{F}_{w}$ of the polyhedron $\mathcal{N}(\phi): \mathcal{F}_{w}:=\{v \in$ $\left.\mathcal{N}(\phi) /\langle w, v\rangle=\inf _{v^{\prime} \in \mathcal{N}(\phi)}\left\langle w, v^{\prime}\right\rangle\right\}$. All faces of the polyhedron $\mathcal{N}(\phi)$ can be recovered in this way. The face of $\mathcal{N}(\phi)$ defined by $w$ is compact if and only if $w$ belongs to the interior $\Delta_{d}$ of the cone $\Delta_{d}$. The cone $\sigma(\mathcal{F}) \subset \Delta_{d}$ associated with the face $\mathcal{F}$ of the polyhedron $\mathcal{N}(\phi)$ is $\sigma(\mathcal{F}):=\left\{u \in \Delta_{d} / \forall v \in\right.$ $\left.\mathcal{F},\langle u, v\rangle=\inf _{v^{\prime} \in \mathcal{N}(\phi)}\left\langle u, v^{\prime}\right\rangle\right\}$. The dual Newton diagram $\Sigma(\mathcal{N}(\phi))$ is the set of cones $\sigma(\mathcal{F})$, for $\mathcal{F}$ running through the set of faces of the polyhedron $\mathcal{N}(\phi)$ (see [Kho77]).

Remark 10. If $\phi=\phi_{1} \cdots \phi_{r}$, the elements of the dual Newton diagram of $\phi$ are the intersections $\bigcap_{i=1}^{r} \sigma_{i}$ for $\sigma_{i}$ running through $\Sigma\left(\mathcal{N}\left(\phi_{i}\right)\right)$ for $i=1, \ldots, r$.

We deduce this property by duality from

$$
\mathcal{N}\left(\phi_{1} \cdots \phi_{r}\right)=\mathcal{N}\left(\phi_{1}\right)+\cdots+\mathcal{N}\left(\phi_{r}\right) .
$$

Definition 11. A polyhedron is polygonal if the maximal dimension of its compact faces is equal to one.

Those Newton polyhedra that are polygonal share some properties of classical Newton polygons of plane curve germs. For instance, any Newton polygon is the Minkowski sum of elementary Newton polygons up to translations (see [Tei80]).

The set of compact faces of a polygonal Newton polyhedron $\mathcal{N}(\phi)$ is combinatorially isomorphic to a finite subdivision of a compact segment: since $\mathcal{N}(\phi)$ is polygonal the cones of the dual Newton diagram that intersect $\stackrel{\Delta}{d}_{d}$ are of dimensions $d$ and $d-1$ by duality.

Lemma 12. If $\phi \in \mathbb{C}\{X\}$ has a polygonal Newton polyhedron, any irreducible factor of $\phi$ that is not associated with $X_{i}$, for $i=1, \ldots, d$, has a polygonal Newton polyhedron.

Proof. It follows from (16) that the compact face of $\mathcal{N}(\phi)$ determined by $w \in \stackrel{\Delta}{d}_{d}$ is the Minkowski sum of the compact faces, determined by $w$, on the Newton polyhedra of the factors. Since the polyhedron $\mathcal{N}(\phi)$ is polygonal the dimension of these compact faces is zero or one. It follows that the Newton polyhedron of an irreducible factor of $\phi$ is polygonal or a translation of $\mathbb{R}_{\geqslant 0}^{d}$, and in the latter case this irreducible factor is associated with one variable.

We give some notation and results for a Weierstrass polynomial $H \in \mathbb{C}\left\{X_{1}, \ldots, X_{d}\right\}[Y]$ to have a polygonal Newton polyhedron. As before we denote $\left(X_{1}, \ldots, X_{d}\right)$ by $X$. We define the Newton polyhedron of a polynomial $H$ as the corresponding Newton polyhedron viewed as a power series. This polyhedron is contained in $\mathbb{R}^{d} \times \mathbb{R}$ with coordinates $(u, v)$. Any irrational vector $w \in \Delta_{d}$, i.e. with linearly independent coordinates over $\mathbb{Q}$, defines a coherent polygonal path on the compact edges of $\mathcal{N}(H)$, (the terminology comes from the combinatorial convexity theory, see [BS92]). This path is defined by $c_{w}(t)=\left(u_{w}(t), t\right)$ where $u_{w}(t)$ is the unique point of the hyperplane section $v=t$ of $\mathcal{N}(H)$ where the minimal value of the linear function $w$ is reached for $t \in\left[\operatorname{ord}_{Y} H, \operatorname{deg} H\right]$ (for $\operatorname{ord}_{Y}$, the order of $H$ as a series in $Y$ ). The point $u_{w}(t)$ is unique because the vertices of the polyhedron $\mathcal{N}(H)$ are rational (they belong to the lattice $\mathbb{Z}^{d} \times \mathbb{Z}$ ). Any compact edge which is not parallel to the hyperplane $v=0$ belongs to some path $c_{w}(t)$ for some irrational vector $w \in \Delta_{d}$. The maximal segments of the polygonal path $c_{w}(t)$ are of the form $\varepsilon_{i}=\left[p_{i}, p_{i+1}\right]$ where $p_{j}=\left(u_{j}, v_{j}\right)$ for $j=i, i+1$ and $v_{i}<v_{i+1}$. We call the vector $q_{i}=\left(u_{i}-u_{i+1}\right) /\left(v_{i+1}-v_{i}\right)$ the inclination and the integer $l_{i}=v_{i+1}-v_{i}$ the height of the edge $\varepsilon_{i}$ (see [Gon00a] where this construction is related to generalizations of the Newton Puiseux Theorem).

Lemma 13. Let $\left\{u_{i}\right\}_{i=1}^{r}$ be $r$ different non zero vectors in $\mathbb{Q}^{d}$ such that $0<u_{r} \leqslant \cdots \leqslant u_{1}$ (with respect to the order (2)) and positive integers $l_{1}, \ldots, l_{r}$. The Minkowski sum

$$
\mathcal{N}=\sum_{i=1}^{r} \frac{l_{i}}{\overline{u_{i}}}
$$




\section{E. R. García Barroso and P. D. González PÉrez}

is a polygonal polyhedron in $\mathbb{R}^{d+1}$. It has $r$ compact edges $\mathcal{E}_{i}$ of inclinations $u_{i}$ and heights $l_{i}$ for $i=1, \ldots, r$. The polyhedron $\mathcal{N}$ determines the terms of the Minkowski sum (17).

Proof. The vector hyperplane $h_{i}$ orthogonal to the compact edge of $\frac{l_{i}}{\overline{u_{i}}}$ defines two half-spaces that subdivide the interior of the cone $\Delta_{d+1}$ since $0<u_{i}$. The condition $u_{i}<u_{j}$ implies that the hyperplanes $h_{i}$ and $h_{j}$ do not intersect in the interior $\Delta_{d+1}$ of the cone $\Delta_{d+1}$. It follows that the possible codimensions of the cones of the dual diagram of $\mathcal{N}$, intersecting $\Delta_{d+1}$, are 0 and 1 . The codimension-one case corresponds to the cones defined by the hyperplane sections $h_{i}$. By duality the polyhedron $\mathcal{N}$ is polygonal. The edge defined by $u \in h_{i} \cap \stackrel{\circ}{\Delta}_{d+1}$ is the Minkowski sum of the faces defined by $u$ on each of the terms of (17), i.e., it is a translation of the polyhedron $\frac{l_{i}}{u_{i}}$.

Lemma 14. Let $H \in \mathbb{C}\{X\}[Y]$ be a Weierstrass polynomial of degree $>0$ with $0 \neq H(0)$ a non-unit. If the polygonal path $c_{w}(t)$ does not depend on the irrational vector of $\omega \in \Delta_{d}$, then the inclinations of the edges of $c_{w}(t)$ are totally ordered with respect to the order (2) and the polyhedron $\mathcal{N}(H)$ is polygonal.

Proof. We label the edges of $c_{w}(t)$ by $\varepsilon_{0}, \ldots, \varepsilon_{r}$ in such a way that $v_{0}=0<v_{1}<\cdots<v_{r}<v_{r+1}=$ $\operatorname{deg} H$ with the previous notation. The irrational vector $w$ defines the total order of $\mathbb{Q}^{d}$ defined by $u \leqslant w u^{\prime} \Leftrightarrow\langle u, w\rangle \leqslant\left\langle u^{\prime}, w\right\rangle$ and we have that

$$
q_{r}<_{w} q_{r-1}<_{w} \cdots<_{w} q_{0}
$$

(see Lemme 5 of [Gon00a]). By hypothesis the path $c_{w}(t)$ does not depend on the irrational $w \in \Delta_{d}$, this implies that the inequality (18) holds for all irrational vectors $w \in \Delta_{d}$, therefore $q_{r} \leqslant q_{r-1} \leqslant$ $\cdots \leqslant q_{0}$ with respect to the order (2). It follows that the polyhedron $\mathcal{N}(H)$ is of the form (17), hence it is polygonal by Lemma 13.

\subsection{A reminder of toric geometry}

We give some definitions and notation (see [Ewa96], [Oda88] or [KKMS73] for proofs). If $N \cong \mathbb{Z}^{d}$ is a lattice, we denote the dual lattice by $M$ and the vector space spanned by $N$ over the field $\mathbb{R}$ (respectively over $\mathbb{Q}$ ) by $N_{\mathbb{R}}$ (respectively $N_{\mathbb{Q}}$ ). In what follows, a cone means a rational convex polyhedral cone: the set of non-negative linear combinations of vectors $a^{1}, \ldots, a^{s} \in N$. The cone $\sigma$ is strictly convex if $\sigma$ contains no linear subspace of dimension greater than zero; the cone $\sigma$ is regular if the primitive integral vectors defining the one-dimensional faces belong to a basis of the lattice $N$. The dual cone $\sigma^{\vee}$ (respectively orthogonal cone $\sigma^{\perp}$ ) of $\sigma$ is the set $\left\{w \in M_{\mathbb{R}} /\langle w, u\rangle \geqslant 0\right.$ (respectively $\langle w, u\rangle=0), \forall u \in \sigma\}$. A fan $\Sigma$ is a family of strictly convex cones in $N_{\mathbb{R}}$ such that any face of such a cone is in the family and the intersection of any two of them is a face of each. The support of the fan $\Sigma$ is the set $\bigcup_{\sigma \in \Sigma} \sigma \subset N_{\mathbb{R}}$. The fan $\Sigma$ is regular if all of its cones are regular. If $\sigma$ is a cone in the fan $\Sigma$ the semigroup $\sigma^{\vee} \cap M$ is of finite type, it spans the lattice $M$ and defines the affine variety $Z^{\sigma^{\vee} \cap M}:=\operatorname{Spec} \mathbb{C}\left[\sigma^{\vee} \cap M\right]$, which we also denote by $Z_{\sigma, N}$, or by $Z_{\sigma}$ when the lattice is clear from the context. If $\sigma \subset \sigma^{\prime}$ are cones in the fan $\Sigma$, then we have an open immersion $Z_{\sigma} \subset Z_{\sigma^{\prime}}$; the affine varieties $Z_{\sigma}$ corresponding to cones in a fan $\Sigma$ glue-up to define the toric variety $Z_{\Sigma}$. The toric variety $Z_{\Sigma}$ is non-singular if and only if the fan $\Sigma$ is regular. The torus, $\left(\mathbb{C}^{*}\right)^{d}$, is embedded as an open dense subset $Z_{\{0\}}$ of $Z_{\Sigma}$, which acts on each chart $Z_{\sigma}$; these actions paste to an action on $Z_{\Sigma}$, which extends the action of the torus on itself by multiplication. The correspondence that associates the Zariski closed subset $\mathbb{O}_{\sigma}$ of $Z_{\sigma}$, defined by the ideal $\left(X^{w} / w \in\left(\sigma^{\vee}-\sigma^{\perp}\right) \cap M\right)$ of $\mathbb{C}\left[\sigma^{\vee} \cap M\right]$, with a cone $\sigma \in \Sigma$ is a bijection between $\Sigma$ and the set of orbits of the torus action in $Z_{\Sigma}$. For example, the set of faces of a cone $\sigma$ defines a fan such that the associated toric variety coincides with $Z_{\sigma}$.

We say that a fan $\Sigma^{\prime}$ is a subdivision of the fan $\Sigma$ if both fans have the same support and if every cone of $\Sigma^{\prime}$ is contained in a cone of $\Sigma$. If $\Sigma^{\prime} \ni \sigma^{\prime} \subset \sigma \in \Sigma$ we have the morphism $Z_{\sigma^{\prime}} \rightarrow Z_{\sigma}$ 
defined by the inclusion of semigroups $\sigma^{\vee} \cap M \rightarrow \sigma^{\vee} \cap M$. These morphisms glue-up and define the toric modification $\pi_{\Sigma^{\prime}}: Z_{\Sigma^{\prime}} \rightarrow Z_{\Sigma}$. Given any fan $\Sigma$ there exists a regular fan $\Sigma^{\prime}$ subdividing $\Sigma$ (see [KKMS73]). The associated toric modification $\pi_{\Sigma^{\prime}}$ is a desingularization.

For instance, the toric variety $Z_{\Delta_{d}}$ associated with the cone $\Delta_{d}:=\left(\mathbb{R}^{d}\right)_{\geqslant 0}^{*}$ and the lattice $N=\left(\mathbb{Z}^{d}\right)^{*}$ is the affine space $\mathbb{C}^{d}$, the orbits correspond to the strata of the stratification defined by the coordinate hyperplanes. Any fan $\Sigma$ supported on $\Delta_{d}$ defines the toric modification $\pi_{\Sigma}: Z_{\Sigma} \rightarrow \mathbb{C}^{d}$. Taking away the cone $\sigma$ from the fan of the cone $\sigma$, geometrically, means taking away the orbit $\mathbb{O}_{\sigma}$ from the variety $Z_{\sigma}$. This implies that the exceptional fiber $\pi_{\Sigma}^{-1}(0)$ of the toric modification $\pi_{\Sigma}$ is

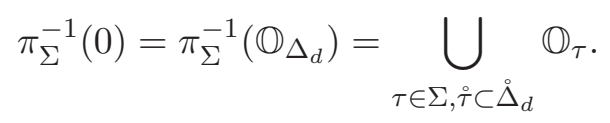

Let $\mathcal{V}$ be a subvariety of $\mathbb{C}^{d}$ such that the intersection with the torus is a dense open subset of $\mathcal{V}$. The strict transform $\mathcal{V}_{\Sigma} \subset Z_{\Sigma}$ is the subvariety of $\pi_{\Sigma}^{-1}(\mathcal{V})$ such that the restriction $\mathcal{V}_{\Sigma} \rightarrow \mathcal{V}$ is a modification. If $0 \neq \phi=\sum c_{a} X^{a} \in \mathbb{C}\{X\}$ is a non-zero series in $X=\left(X_{1}, \ldots, X_{d}\right)$ the dual Newton diagram $\Sigma(\mathcal{N}(\phi))$ is a subdivision of $\Delta_{d}$. The symbolic restriction of $\phi$ to a set $\mathcal{F} \subset M_{\mathbb{R}}$ is $\phi_{\mid \mathcal{F}}:=\sum_{a \in \mathcal{F}} c_{a} X^{a}$.

Lemma 15. Let $\phi=\sum c_{a} X^{a} \in \mathbb{C}\left\{X_{1}, \ldots, X_{d}\right\}$ be an irreducible series, not associated with any of the variables $X_{1}, \ldots, X_{d}$, defining the germ $(\mathcal{V}, 0) \subset\left(\mathbb{C}^{d}, 0\right)$. Let $\Sigma$ be any subdivision of the dual Newton diagram $\Sigma(\mathcal{N}(\phi))$. If $\sigma \in \Sigma$ and if $\stackrel{\circ}{\sigma} \subset \stackrel{\circ}{\Delta}_{d}$, the intersection $\mathbb{O}_{\sigma} \cap \mathcal{V}_{\Sigma}$ is defined by the vanishing of $X^{-u} \phi_{\mathcal{F}_{\sigma}} \in \mathbb{C}\left[\sigma^{\perp} \cap M\right]$ where $u$ is any vertex of the compact face $\mathcal{F}_{\sigma}$ of $\mathcal{N}(\phi)$ defined by any $u \in \stackrel{\circ}{\sigma}$.

Proof. Let $v \in M$ such that $-v+\mathcal{N}(\phi) \subset \sigma^{\vee}$. Then all the terms in $X^{-v} \phi$ vanish on the orbit $\mathbb{O}_{\sigma}$ (since their exponents belong to $\sigma^{\vee}-\sigma^{\perp}$ ) unless the vector $v$ belongs to the affine hull $\operatorname{Aff}\left(\mathcal{F}_{\sigma}\right)$ of the compact face $\mathcal{F}_{\sigma}$. In this case we have that $\left(X^{-v} \phi\right) \mid \mathbb{O}_{\sigma}=X^{-v} \phi_{\mid \mathcal{F}_{\sigma}}$. If $v, v^{\prime} \in \operatorname{Aff}\left(\mathcal{F}_{\sigma}\right) \cap M$ we have that $v-v^{\prime}$ belongs to $\sigma^{\perp} \cap M$, therefore the polynomials $X^{-v} \phi_{\mid \mathcal{F}_{\sigma}}$ and $X^{-v^{\prime}} \phi_{\mid \mathcal{F}_{\sigma}}$ are related by the invertible function $X^{v-v^{\prime}}$ on the torus $\mathbb{O}_{\sigma}$. It follows from this that $X^{-v} \phi_{\mid \mathcal{F}_{\sigma}}$ defines the ideal of the intersection $\mathcal{V}_{\Sigma} \cap \mathbb{O}_{\sigma}$.

\subsection{An irreducibility criterion for series with polygonal Newton polyhedra}

We use Theorem 1 to prove Theorem 2 by translating the existence of the bunch decomposition of $f_{Y}$ in geometrical terms by means of an irreducibility criterion for power series with polygonal Newton polyhedron. The criterion, which holds when the field $\mathbb{C}$ of complex numbers is replaced by an algebraically closed field of arbitrary characteristic, generalizes a fundamental property of plane curves. We introduce some definitions and notation.

Let $\mathcal{E}=\left[\alpha, \alpha^{\prime}\right]$ be a compact segment joining two elements $\alpha, \alpha^{\prime}$ of the lattice $\mathbb{Z}^{d}$, and denote by $u$ the primitive integral vector parallel to $\mathcal{E}$, i.e. we have an equality of the form $\alpha^{\prime}-\alpha=l u$ for a maximal integer $l \geqslant 1$. If $\phi=\sum c_{a} X^{a}$ is a series in $\mathbb{C}\left\{X_{1}, \ldots, X_{d}\right\}$ we have that

$$
X^{-\alpha}\left(\sum_{a \in \mathcal{E} \cap \mathbb{Z}^{d}} c_{a} X^{a}\right)=\sum_{i=0}^{l} c_{i q} X^{i u}=p(\mathcal{E}, \phi)\left(X^{u}\right)
$$

where $p(\phi, \mathcal{E})$ is the polynomial $p(\phi, \mathcal{E})=\sum_{i=0}^{l} c_{i q} t^{i}$. Obviously, this definition depends on the order of the vertices: the polynomial obtained by interchanging the vertices $\alpha$ and $\alpha^{\prime}$ of $\mathcal{E}$ is equal to $t^{l} p(\phi, \mathcal{E})\left(t^{-1}\right)$. In both cases these two polynomials define isomorphic subschemas of $\mathbb{C}^{*}$. If, in addition, $\phi$ is a polynomial in $\mathbb{C}\left\{X_{1}, \ldots, X_{d-1}\right\}\left[X_{d}\right]$ and if we can fix the order of the vertices $\alpha=(a, t)$ and $\left(a^{\prime}, t^{\prime}\right)$ of $\mathcal{E}$ by the convention $t<t^{\prime}$, we obtain that the polynomial $p(\phi, \mathcal{E})$ is defined in a unique way by $(20)$. 


\section{E. R. García Barroso and P. D. González PÉrez}

Theorem 3. If $\phi \in \mathbb{C}\left\{X_{1}, \ldots, X_{d}\right\}$ is irreducible and has a polygonal Newton polyhedron $\mathcal{N}(\phi)$, then the polyhedron $\mathcal{N}(\phi)$ has only one compact edge $\mathcal{E}$ and the polynomial $p(\phi, \mathcal{E})$ has only one root in $\mathbb{C}^{*}$, counted without multiplicity.

Proof. Since $\mathcal{N}(\phi)$ is polygonal the cones $\sigma$ in the dual Newton diagram $\Sigma$ of the polyhedron $\mathcal{N}(\phi)$, such that $\stackrel{\circ}{\sigma} \subset \Delta_{d}$ are of codimensions 0 or 1 (the possible dimensions of the compact faces of $\mathcal{N}(\phi)$. We keep notation of Lemma 15. If $\operatorname{dim} \sigma=d$ it follows from Lemma 15 that the intersection $\mathcal{V}_{\Sigma} \cap \mathbb{O}_{\sigma}$ is empty. If $\operatorname{dim} \sigma=d-1$ the cone $\sigma$ corresponds to the compact edge $\mathcal{E}$ of the polyhedron $\mathcal{N}(\phi)$. By Lemma 15 the intersection $\mathcal{V}_{\Sigma} \cap \mathbb{O}_{\sigma}$ is defined by the vanishing of $X^{-u} \phi_{\mathcal{E}}$ on the torus $\mathbb{O}_{\sigma}$ (the vector $u$ being one of the vertices of the edge $\mathcal{E}$ ). We have that the coordinate ring $\mathbb{C}\left[\sigma^{\perp} \cap M\right]$ of the orbit $\mathbb{O}_{\sigma} \cong \mathbb{C}^{*}$ is isomorphic to $\mathbb{C}\left[X^{ \pm u}\right]$, where $u$ is the primitive integral vector parallel to the edge $\mathcal{E}$. By formula (20) the polynomial $X^{-u} \phi_{\mathcal{E}}$ corresponds to the polynomial $p(\mathcal{E}, \phi)\left(X^{u}\right)$. It follows that the intersection $\mathcal{V}_{\Sigma} \cap \mathbb{O}_{\sigma}$ is non-empty. It is a finite set of points counted with multiplicities that correspond to the zeros of the polynomial $p(\mathcal{E}, \phi)$. By (19) the fiber of the modification $\pi_{\Sigma} \mid \mathcal{V}_{\Sigma}: \mathcal{V}_{\Sigma} \rightarrow \mathcal{V}$ is equal to the discrete set $\bigcup\left(\mathbb{O}_{\sigma} \cap \mathcal{V}_{\Sigma}\right)$, where $\sigma$ runs through the cones $\sigma \in \Sigma$ such that $\stackrel{\sigma}{\subset} \stackrel{\circ}{\Delta}_{d}$. Since by hypothesis the germ $\mathcal{V}$ is analytically irreducible at the origin, this fiber is a connected set by Zariski's Main Theorem (see [Mum88, Zar55]), thus it is reduced to one point. This implies that the Newton polyhedron $\mathcal{N}(\phi)$ has only one compact edge $\mathcal{E}$ and that the polynomial $p(\mathcal{E}, \phi)$ has only one root in $\mathbb{C}^{*}$ (counted without multiplicity).

We will need the following lemma in $\S 8$. We denote by $M$ (respectively by $M^{\prime}$ ) the lattice spanned by the exponents of monomials in $\mathbb{C}\left\{X_{1}, \ldots, X_{d}\right\}$ (respectively in $\mathbb{C}\left\{X_{1}, \ldots, X_{d}, Y\right\}$ ).

Lemma 16. Let $h \in \mathbb{C}\left\{X_{1}, \ldots, X_{d}\right\}[Y]$ be a monic polynomial, and $\lambda \in M_{\mathbb{Q}} \cap \Delta_{d}^{\vee}$. We denote the compact edge of the polyhedron $\operatorname{deg} h / / \lambda$ by $\mathcal{E}$. If $\mathcal{N}(h) \subset \operatorname{deg} h / / \lambda$ and if $p(\mathcal{E}, h)=t^{\operatorname{deg} h}$, the strict transform of $h=0$ by $\pi_{\Sigma}$, for $\Sigma=\Sigma(\operatorname{deg} h / / \lambda)$, intersects $\pi_{\Sigma}^{-1}(0)$ only at the zero-dimensional orbit $\mathbb{O}_{\tau}$ where $\tau$ is the cone associated with the vertex $((\operatorname{deg} h) \lambda, 0)$ of the polyhedron $\operatorname{deg} h / / \lambda$.

Proof. Denote by $v$ the vertex $(0, \operatorname{deg} h)$ of $\operatorname{deg} h / / \lambda$. We deduce from the hypothesis $\mathcal{N}(h) \subset \operatorname{deg} h / / \lambda$ and $p(\mathcal{E}, h)=t^{\operatorname{deg} h}$ that the series $Y^{-\operatorname{deg} h} h$ has terms in $\mathbb{C}\left[\sigma^{\vee} \cap M^{\prime}\right]$, its constant term is equal to one, and the other terms have exponents in $\sigma^{\vee}-\sigma^{\perp}$, for $\sigma=\sigma(\{v\})$ or $\sigma=\sigma(\mathcal{E})$. It follows from Lemma 15, that the strict transform of $h=0$ does not meet $\mathbb{O}_{\sigma}$ since the terms $\neq 1$ vanish on $\mathbb{O}_{\sigma}$. We deduce from (19) that the strict transform of $h$ intersects the exceptional fiber $\pi_{\Sigma}^{-1}(0)$ at the closed orbit $\mathbb{O}_{\tau}$ (which is reduced to a point since $\tau$ is of maximal dimension $d+1$ ).

\section{Type of the decomposition and Newton polyhedra of images}

We apply the irreducibility criterion to clarify the relation between the type of the $f$-bunch decomposition of $h \in \mathcal{C}_{f}$ and the Newton polyhedra $\mathcal{N}\left(\psi_{f_{i}}(h)\right)$.

Lemma 17. The restriction of the valuation $\nu_{i}$ to $\theta_{f h}\left(f_{i}\right)$ is an order-preserving bijection. The characteristic exponents of $f_{i}$ and the valuation $\nu_{i}\left(P_{\lambda}^{(i)}\right)$ determine $\lambda$.

Proof. We denote the characteristic exponents of $f_{i}$ by $\lambda_{1}, \ldots, \lambda_{g}$. Let $P_{\lambda}$ be a vertex of $\theta_{f h}\left(f_{i}\right)$. For simplicity we drop the index $i$. If $\lambda<\lambda_{1}$ then we have that $\nu\left(P_{\lambda}\right)=n \lambda<\nu\left(P_{\lambda_{1}}\right)=n \lambda_{1}$. Otherwise there exists a unique $1 \leqslant j \leqslant g$ such that $\lambda_{j} \leqslant \lambda<\lambda_{j+1}$ since $V_{f h}\left(f_{i}\right)$ is totally ordered 


\section{DECOMPOSITION IN BUNCHES OF THE CRITICAL LOCUS OF A QUASI-ORDINARY MAP}

by Lemma 1. The first assertion follows from the inequality

$$
\begin{aligned}
\nu\left(P_{\lambda_{j}}\right) & =e_{j-1} \lambda_{j}+\sum_{k=1}^{j-1}\left(e_{k-1}-e_{k}\right) \lambda_{k}=e_{j} \lambda_{j}+\sum_{k=1}^{j}\left(e_{k-1}-e_{k}\right) \lambda_{k} \\
& \leqslant e_{j} \lambda+\sum_{k=1}^{j}\left(e_{k-1}-e_{k}\right) \lambda_{k}=\nu\left(P_{\lambda}\right)<e_{j} \lambda_{j+1}+\sum_{k=1}^{j}\left(e_{k-1}-e_{k}\right) \lambda_{k}=\nu\left(P_{\lambda_{j+1}}\right) .
\end{aligned}
$$

If we know the characteristic exponents of $f_{i}$, then there is a unique $j$ such that $\nu\left(P_{\lambda_{j}}\right) \leqslant \nu\left(P_{\lambda}\right)<$ $\nu\left(P_{\lambda_{j+1}}\right)$ where $\lambda_{0}:=0$. Then we recover $\lambda$ from (11).

If $h \in \mathcal{R} C_{f}$ we show that the Newton polyhedron of $\psi_{f_{i}}(h)$ is determined by the $f$-type of $h$.

Proposition 18. If $\left\{\left(q_{1, r}, \ldots, q_{s, r} ; c_{r}\right)\right\}_{r=1}^{s(f, h)}$ is the type of the $f$-bunch decomposition of a polynomial $h \in \mathcal{R} C_{f}$, then the Newton polyhedron of $\psi_{f_{i}}(h)$ is the Minkowski sum

$$
\mathcal{N}\left(\psi_{f_{i}}(h)\right)=\sum_{r=1}^{s(f, h)} \frac{c_{r}}{\overline{q_{i, r}}} .
$$

Proof. Let $\left\{\tau_{r}^{(j)}\right\}_{j=1, \ldots, \operatorname{deg} b_{r}}$ be the roots of the factor $b_{r}$ of the $f$-bunch decomposition of $h$, for $r=1, \ldots, s(f, h)$, viewed in some suitable ring extension of the form $\mathbb{C}\left\{X^{1 / k}\right\}$. It follows from Proposition 5 and the definition of the bunches that:

$$
f_{i}\left(\tau_{r}^{(j)}\right)=X^{q_{i, r}} \epsilon_{i, r, j}, \quad \text { where } \epsilon_{i, r, j} \text { is a unit in } \mathbb{C}\left\{X^{1 / k}\right\} .
$$

By general properties of the resultant we have that: $\psi_{f}\left(b_{r}\right)=\prod_{j=1}^{\operatorname{deg} b_{r}}\left(T-f\left(\tau_{r}^{(j)}\right)\right)$. We deduce from this and (22) that the polyhedron $\mathcal{N}\left(\psi_{f_{i}}\left(b_{r}\right)\right)=\sum_{j=1}^{c_{r}} \mathcal{N}\left(T-f_{i}\left(\tau_{r}^{(j)}\right)\right)$ is equal to $c_{r} / / q_{i, r}$ and equality (21) follows from the property (16).

Remark 19. If $h \in \mathcal{C}_{f}$ the assertion of Proposition 18 is not true in general.

For instance, if $f=Y$ we have that $\mathcal{C}_{f}$ is the set of monic polynomials $h \in \mathbb{C}\{X\}[Y]$ such that $h(0)$ is of the form $h(0)=X^{\rho(f, h)} \epsilon_{f, h}$ with $\epsilon_{f, h}(0) \neq 0$. On the other hand $\mathcal{R} C_{f}$ is the set of monic polynomials $h \in \mathbb{C}\{X\}[Y]$ such that the product $Y h$ is a quasi-ordinary polynomial. If $f=Y$ and if $h(Y) \in \mathcal{C}_{f}$ the polynomial $\psi_{f}(h(Y))$ is equal to $h(T)$. The polynomial $h(Y)=$ $Y^{2}+\left(X_{1}+X_{2}\right) Y+X_{1} X_{2}^{2}$ belongs to $\mathcal{C}_{f} \backslash \mathcal{R} C_{f}$ and the Newton polyhedron of $\psi_{f}(h)=h(T)$ is not polygonal.

The following proposition generalizes Proposition 3.4.8 [Pop01].

Proposition 20. Given $\theta_{f}(f)$, if $h \in \mathcal{R} C_{f}$ the following informations determine each other.

(i) The contact chain $[h]^{(f)}$.

(ii) The type of the bunch decomposition of $h$ induced by $f$.

(iii) The collection of Newton polyhedra of $\psi_{f_{i}}(h)$ for $i=1, \ldots, s$.

Proof. (i) $\Rightarrow$ (ii) If the contact chain is $[h]^{(f)}=\sum c_{i} P_{i}$ the type of the $f$-bunch decomposition of $h$ is $\left\{\left(\nu_{1}\left(P_{i}\right), \ldots, \nu_{s}\left(P_{i}\right) ; c_{i}\right)\right\}_{i}$ by Remark 7 . The implication (ii) $\Rightarrow$ (iii) is a direct consequence of Proposition 18. (iii) $\Rightarrow$ (i) The Newton polyhedron of $\psi_{f_{i}}(h)$ is polygonal by Proposition 18 . We recover the set of vertices $\left\{P_{j}^{(i)}\right\}_{j}$ of $\theta_{f h}\left(f_{i}\right)$ corresponding to the orders of coincidence of $f_{i}$ with the irreducible factors of $h$ from the inclinations of the compact edges by Lemma 17 (since $\theta_{f}\left(f_{i}\right)$ is given): the maximal point $P_{j_{0}}^{(i)}$ of the set $\left\{P_{j}^{(i)}\right\}_{j}$ corresponds to a factor $b_{1}$ of the $f$-bunch decomposition of degree $c_{1}$ equal to the height of the edge of $\mathcal{N}\left(\psi_{f_{i}}(h)\right)$ of maximal inclination $\nu_{i}\left(P_{j_{0}}^{(i)}\right)$. 


\section{E. R. García Barroso and P. D. González Pérez}

Then we can replace $h$ by $h^{\prime}=h / b_{1}$ and continue in the same way. The Newton polyhedra of $\psi_{f_{i}}\left(h^{\prime}\right)$ is obtained from $\mathcal{N}\left(\psi_{f_{i}}\left(h^{\prime}\right)\right)$ by subtracting the elementary polyhedra $c_{1} / / \nu_{i}\left(P_{j_{0}}^{(i)}\right)$ (the subtraction makes sense by Lemma 13).

Proposition 21. Given $\theta_{f}(f)$ and $h \in \mathcal{C}_{f}$, if the Newton polyhedra $\psi_{f_{i}}(h)$ for $i=1, \ldots, s$, are polygonal the following pieces of information determine each other.

(i) The type of the bunch decomposition of $h$ induced by $f$.

(ii) The collection of Newton polyhedra of $\psi_{f_{i}}(h)$ for $i=1, \ldots, s$.

Proof. If $\operatorname{deg} h=m>0$, the type of $h$ determines two vertices of $\mathcal{N}\left(\psi_{f_{i}}(h)\right)$ : the vertex $(0, m)$ that corresponds to the monomial $T^{m}$ and the vertex $\left(\rho\left(f_{i}, h\right), 0\right)$ that corresponds to the dominant term of $\left(\psi_{f_{i}}(h)\right)_{\mid T=0}=\operatorname{Res}_{Y}\left(-f_{i}, h\right)$. In particular, if $h$ is irreducible and $\psi_{f_{i}}(h)$ has a polygonal Newton polyhedron, then $\psi_{f_{i}}(h)$ is irreducible. By the irreducibility criterion, $\mathcal{N}\left(\psi_{f_{i}}(h)\right)$ has only one compact edge equal to $\left[(0, m),\left(\rho\left(f_{i}, h\right), 0\right)\right]$. We obtain that

$$
\mathcal{N}\left(\psi_{f_{i}}(h)\right)=\frac{\operatorname{deg} h}{\overline{\rho\left(f_{i}, h\right) / \operatorname{deg} h}} .
$$

If $h^{\prime}$ and $h^{\prime \prime}$ are two monic irreducible factors of $h$ and if $h^{\prime} \sim_{f} h^{\prime \prime}$ then $\rho\left(f_{i}, h^{\prime}\right) / \operatorname{deg} h^{\prime}=\rho\left(f_{i}, h^{\prime \prime}\right) /$ $\operatorname{deg} h^{\prime \prime}$ for $i=1, \ldots, s$. We show using (16) and (13) that

$$
\begin{aligned}
\mathcal{N}\left(\psi_{f_{i}}\left(h^{\prime} h^{\prime \prime}\right)\right) & =\mathcal{N}\left(\psi_{f_{i}}\left(h^{\prime}\right) \psi_{f_{i}}\left(h^{\prime \prime}\right)\right)=\mathcal{N}\left(\psi_{f_{i}}\left(h^{\prime}\right)\right)+\mathcal{N}\left(\psi_{f_{i}}\left(h^{\prime \prime}\right)\right) \\
& =\frac{\operatorname{deg} h^{\prime}}{\overline{\rho\left(f_{i}, h^{\prime}\right) / \operatorname{deg} h^{\prime}}}+\frac{\operatorname{deg} h^{\prime \prime}}{\overline{\rho\left(f_{i}, h^{\prime \prime}\right) / \operatorname{deg} h^{\prime \prime}}}=\frac{\operatorname{deg}\left(h^{\prime} h^{\prime \prime}\right)}{\overline{\rho\left(f_{i}, h^{\prime}\right) / \operatorname{deg} h^{\prime}}} .
\end{aligned}
$$

If $h=b_{1} \cdots b_{s(f, h)}$ is the $f$-bunch factorization of $h$, and if $h_{j}$ denotes any irreducible factor of the polynomial $b_{j}$, for $j=1, \ldots, s(f, h)$. We deduce that $\mathcal{N}\left(\psi_{f_{i}}(h)\right)$ is the Minkowski sum

$$
\mathcal{N}\left(\psi_{f_{i}}(h)\right)=\sum_{j=1}^{s(f, h)} \mathcal{N}\left(\psi_{f_{i}}\left(b_{j}\right)\right)=\sum_{j=1}^{s(f, h)} \frac{\operatorname{deg} b_{j}}{\overline{\rho\left(f_{i}, h_{j}\right) / \operatorname{deg} h_{j}}} .
$$

Conversely, if we are given the tree $\theta_{f}(f)$ and polygonal polyhedron $\mathcal{N}\left(\psi_{f_{i}}(h)\right)$, for $i=1, \ldots, s$, we recover the type from the inclinations and heights of the compact edges of $\mathcal{N}\left(\psi_{f_{i}}(h)\right)$ following the method of Proposition 20.

LEmma 22. If $f_{Y} \in \mathcal{R} C_{f}$, then the Newton polyhedra of $\psi_{f_{i}}\left(f_{Y}\right)$ are polygonal, for $i=1, \ldots, s$.

Proof. If $f_{Y} \in \mathcal{R} C_{f}$, the type of $f_{Y}$ is given in terms of the Eggers-Wall tree by formula (15) by applying the Popescu-Pampu decomposition (see Remark 9). Then we obtain the polyhedra $\psi_{f_{i}}\left(f_{Y}\right)$ for $i=1, \ldots, s$, from the type of $f_{Y}$ by formula (21). These polyhedra are polygonal by Lemmas 17 and 13 .

\section{Toric base changes and the Eggers-Wall tree}

Consider a germ of toric morphism $\left(\mathbb{C}^{d}, 0\right) \rightarrow\left(\mathbb{C}^{d}, 0\right)$ defined in suitable coordinates by

$$
\begin{aligned}
X_{1} & =V_{1}^{a_{1}^{(1)}} V_{2}^{a_{1}^{(2)}} \cdots V_{d}^{a_{1}^{(d)}} \\
X_{2} & =V_{1}^{a_{2}^{(1)}} V_{2}^{a_{2}^{(2)}} \cdots V_{d}^{a_{2}^{(d)}} \\
& \vdots \\
X_{d} & =V_{1}^{a_{d}^{(1)}} V_{2}^{a_{d}^{(2)}} \cdots V_{d}^{a_{d}^{(d)}}
\end{aligned}
$$




\section{DECOMPOSITION IN BUNCHES OF THE CRITICAL LOCUS OF A QUASI-ORDINARY MAP}

where the vectors $a^{(i)}:=\left(a_{1}^{(i)}, \ldots, a_{d}^{(i)}\right)$, for $i=1, \ldots, d$, span a regular cone $\tau$ contained in $\Delta_{d}=$ $\left(\mathbb{R}^{d}\right)_{\geqslant 0}^{*}$, and define a basis of the lattice $N$ dual to the lattice $M$ of exponents of Laurent monomials in $X_{1}, \ldots, X_{d}$. We denote by $H^{(\tau)}$ the image of a polynomial $H \in \mathbb{C}\left\{X_{1}, \ldots, X_{d}\right\}[Y]$ in the ring $\mathbb{C}\left\{V_{1}, \ldots, V_{d}\right\}[Y]$ by the base change $(23)$. If $f \in \mathbb{C}\left\{X_{1}, \ldots, X_{d}\right\}[Y]$ is a quasi-ordinary polynomial so is $f^{(\tau)}$ since $\Delta_{Y}\left(f^{(\tau)}\right)$ is the image of $\Delta_{Y}(f)$ by $(23)$, and this toric morphism transforms monomials into monomials. We reformulate the definition of the Eggers-Wall tree in such a way that the relation between the trees associated with $f$ and $f^{(\tau)}$ is clarified. The main idea has already been used in [Gon00b, Gon00a].

The ring of convergent (or formal) complex power series in $X=\left(X_{1}, \ldots, X_{d}\right)$ can be denoted by $\mathbb{C}\left\{\Delta_{d}^{\vee} \cap M\right\}$. The toric morphism (23) corresponds to the local ring extension $\mathbb{C}\left\{\Delta_{d}^{\vee} \cap M\right\} \rightarrow$ $\mathbb{C}\left\{\tau^{\vee} \cap M\right\}$ induced by the semigroup inclusion $\Delta_{d}^{\vee} \cap M \subset \tau^{\vee} \cap M$ (the dual cone $\tau^{\vee}$ contains $\Delta_{d}^{\vee}$ since $\tau \subset \Delta_{d}$ ). The monomial $X_{1}^{u_{1}} \cdots X_{d}^{u_{d}} \in \mathbb{C}\left\{\Delta_{d}^{\vee} \cap M\right\}$ is determined by an element $u$ of the lattice $M$ and the regular cone $\Delta_{d}^{\vee}$. The $d$-uplet $\left(u_{1}, \ldots, u_{d}\right)$ defines the coordinates of $u$ with respect to the basis of $M$ defined by the regular cone $\Delta_{d}^{\vee}$ (this basis is uniquely determined as a set). The vector $u \in M$ remains unchanged by the semi-group inclusion $\Delta_{d}^{\vee} \cap M \subset \tau^{\vee} \cap M$ and thus the image of the monomial $X_{1}^{u_{1}} \ldots X_{d}^{u_{d}}$ by (23) is described in the same way in terms of the vector $u \in M$ and the cone $\tau^{\vee}$.

Let $f \in \mathbb{C}\left\{X_{1}, \ldots, X_{d}\right\}[Y]$ be a quasi-ordinary polynomial. If $P$ is a non-extremal vertex of the elementary branch $\theta_{f}\left(f_{i}\right)$, the valuation $v(P)=\left(\lambda_{1}, \ldots, \lambda_{d}\right)$ defines the coordinates of an element $\tilde{v}(P)$ of the characteristic lattice $M_{g(i)}^{(i)} \subset M_{\mathbb{Q}}$ associated with $f_{i}$, with respect to the basis of $M$ defined by the regular cone $\Delta_{d}^{\vee}$. Conversely, the element $\tilde{v}(P)$ and the basis of $M$ associated with the regular cone $\Delta_{d}^{\vee}$ determine $v(P)$. If $v(P)=+\infty$ we set $\tilde{v}(P)=+\infty$. We define in this way a lattice valuation $\tilde{v}$ of the vertices of $\theta_{f}(f)$ with images in the vector space $M_{\mathbb{Q}}$ (considered as an abstract vector space). We deduce the following lemma.

Lemma 23. The Eggers-Wall tree of the quasi-ordinary polynomial $f \in \mathbb{C}\left\{\Delta_{d}^{\vee} \cap M\right\}[Y]$, determines and is determined by the simplicial complex $\theta_{f}(f)$, the chain $\gamma_{f}$, the lattice valuation $\tilde{v}$ and the reference lattice cone $\left(\Delta_{d}^{\vee}, M\right)$.

We use this idea to describe the relations between the Eggers-Wall tree of $f$ and $f^{(\tau)}$.

Lemma 24. The simplicial complex $\theta_{f^{(\tau)}}\left(f^{(\tau)}\right)$, the chain $\gamma_{f^{(\tau)}}$ and the lattice valuation $\tilde{v}^{(\tau)}$ associated with the quasi-ordinary polynomial $f^{(\tau)}$ coincide with those associated with $f$. The reference lattice cone associated with $f^{(\tau)}$ is equal to $\left(\tau^{\vee}, M\right)$.

Proof. By the Jung-Abhyankar theorem, there exists an integer $k>0$ such that all the roots of $f$ belong to $\mathbb{C}\left\{\Delta_{d}^{\vee} \cap k^{-1} M\right\}$. If $\zeta^{(i)} \in \mathbb{C}\left\{\Delta_{d}^{\vee} \cap k^{-1} M\right\}$ is a root of $f$, then $\left(\zeta^{(i)}\right)^{(\tau)} \in \mathbb{C}\left\{\tau^{\vee} \cap k^{-1} M\right\}$ is a root of $f^{(\tau)}$. Extending the cone does not modify the support of the series nor the lattices spanned by the exponents. It then follows from Lipman's characterization of roots of quasi-ordinary polynomials that $\zeta^{(i)}$ and $\left(\zeta^{(i)}\right)^{(\tau)}$ have characteristic exponents defined by the same elements of the lattice $k^{-1} M$, and that if $f$ is irreducible the same holds for $f^{(\tau)}$ (see [Lip83, Proposition 1.5] or [Gau88, Proposition 1.3]). Then the equality $\tilde{\theta}_{f}(f)=\tilde{\theta}_{f(\tau)}\left(f^{(\tau)}\right)$ follows from the fact that the ring extension $\mathbb{C}\left\{\Delta_{d}^{\vee} \cap k^{-1} M\right\} \hookrightarrow \mathbb{C}\left\{\tau^{\vee} \cap k^{-1} M\right\}$ sends monomials to monomials and units to units.

We consider Newton polyhedra of $H \in \mathbb{C}\left\{X_{1}, \ldots, X_{d}\right\}[Y]$ and $H^{(\tau)} \in \mathbb{C}\left\{U_{1}, \ldots, U_{d}\right\}[Y]$ in the same affine space.

Remark 25. Let $\phi \in\left\{\Delta_{d}^{\vee} \cap M\right\}$ and $H \in \mathbb{C}\left\{\Delta_{d}^{\vee} \cap M\right\}[Y]$ be non-zero. We have that

$$
\mathcal{N}\left(\phi^{(\tau)}\right)=\mathcal{N}(\phi)+\tau^{\vee} \text { and } \mathcal{N}\left(H^{(\tau)}\right)=\mathcal{N}(H)+\left(\tau^{\vee} \times \mathbb{R}_{\geqslant 0}\right) .
$$




\section{E. R. García Barroso and P. D. González Pérez}

Lemma 26. If $\tau \subset \Delta_{d}$ is a regular cone the coherent polygonal paths defined by an irrational vector $w \in \tau$ on the edges of the polyhedra $\mathcal{N}(F)$ and $\mathcal{N}\left(F^{(\tau)}\right)$ coincide.

\section{The proofs of the results on the type of $f_{Y}$}

Proof of Theorem 1. We first discuss the case of the Newton polyhedron of $\psi_{f_{i}}\left(f_{Y}\right)$. If $f_{Y} \notin \mathcal{R} C_{f}$, let $\Sigma$ be a regular subdivision of the dual Newton diagram of $\Delta_{Y}\left(f_{Y} \cdot f\right)$. Let $\tau \in \Sigma$ be a cone of dimension $d$. By construction of the fan $\Sigma$, the image $\left(\Delta_{Y}\left(f_{Y} \cdot f\right)\right)^{(\tau)}$ of the discriminant $\Delta_{Y}\left(f_{Y} \cdot f\right)$ in the ring $\mathbb{C}\left\{\tau^{\vee} \cap M\right\}$ is of the form a monomial times a unit (see the proof of Lemma 15). Since $\left(\Delta_{Y}\left(f_{Y} \cdot f\right)\right)^{(\tau)}=\Delta_{Y}\left(f_{Y} \cdot f\right)^{(\tau)}$ the polynomial $\left(f^{(\tau)}\right)_{Y}=\left(f_{Y}\right)^{(\tau)}$ belongs to $\mathcal{R} C_{f(\tau)}$. By Lemma 22 applied to $f^{(\tau)}$, the polyhedron $\mathcal{N}\left(\psi_{f_{i}^{(\tau)}}\left(f_{Y}^{(\tau)}\right)\right)$ is polygonal and defined by (14) (with respect to the lattice cone $\left.\left(\tau^{\vee}, M\right)\right)$. The union of its compact faces is a polygonal path $c^{(\tau)}$ in the affine space $\mathbb{R}^{d+1}$, and we have that $\mathcal{N}\left(\psi_{f_{i}^{(\tau)}}\left(f_{Y}^{(\tau)}\right)\right)=c^{(\tau)}+\tau^{\vee} \times \mathbb{R}_{\geqslant 0}$. It follows from Lemma 22 that the polygonal path $c^{(\tau)}$ is determined by the simplicial complex $\theta_{f(\tau)}\left(f^{(\tau)}\right)$, the chain $\gamma_{f(\tau)}$ and the lattice valuation $\tilde{v}^{(\tau)}$. By Lemma 24, we have that $\theta_{f(\tau)}\left(f^{(\tau)}\right)=\theta_{f}(f), \gamma_{f(\tau)}=\gamma_{f}$ and $\tilde{v}^{(\tau)}=\tilde{v}$ hence the polygonal path $c^{(\tau)}$ does not depend on $\tau$. We denote $c^{(\tau)}$ by $c$. We deduce from this and Remark 26 that the coherent polygonal path defined by an irrational vector $w \in \Delta_{d}$ on the polyhedron $\mathcal{N}\left(\psi_{f_{i}}\left(f_{Y}\right)\right)$ is equal to $c$. It follows from Lemma 14 that $\mathcal{N}\left(\psi_{f_{i}}\left(f_{Y}\right)\right)=c+\Delta_{d}^{\vee} \times \mathbb{R}_{\geqslant 0}$. Therefore, the Newton polyhedron of $\psi_{f_{i}}\left(f_{Y}\right)$ is polygonal and defined by (14).

The case of the Newton polyhedron of $\psi_{f}\left(f_{Y}\right)$ is discussed analogously, if $f_{Y} \in \mathcal{R} C_{f}$ and $f=$ $b_{1} \ldots b_{s\left(f, f_{Y}\right)}$ is its bunch decomposition the result follows: the Minkowski sum (14) corresponds to the decomposition $\psi_{f}\left(f_{Y}\right)=\prod_{i=1}^{s\left(f, f_{Y}\right)} \operatorname{Res}_{Y}\left(T-f_{1} \cdots f_{s}, b_{i}\right)$.

Remark 27. The Newton polyhedron of $\psi_{f}\left(f_{Y}\right)$ is not necessarily polygonal since the set $\{v(P)\}$, for $P$ running through the non-extremal vertices of $\theta_{f}(f)$ is not totally ordered in general.

Proof of Theorem 2. By Theorem 1 the Newton polyhedra $\psi_{f_{i}}\left(f_{Y}\right)$, for $i=1, \ldots, s$, are polygonal and coincide with the those obtained assuming the hypothesis of $f_{Y} \in \mathcal{R} C_{f}$. Then assertion (i) follows by Proposition 21.

To prove assertion (ii) we consider the matrix $\mathcal{M}=\left(m_{i, j}\right)$ whose columns are the $(s+1)$-uples of vectors defining the type of $f_{Y}$ :

$$
\left(\frac{\rho\left(f_{1}, h_{j}\right)}{\operatorname{deg} h_{j}}, \ldots, \frac{\rho\left(f_{s}, h_{j}\right)}{\operatorname{deg} h_{j}} ; \operatorname{deg} b_{j}\right) \quad \text { for } j=1, \ldots, s\left(f, f_{Y}\right) .
$$

By definition, the columns of the matrix $\mathcal{M}$ correspond bijectively to the bunches of the decomposition of $f_{Y}$ induced by $f$. By Theorem 2 these bunches correspond bijectively with the non-extremal vertices of the tree $\theta_{f}(f)$, in such a way that if the column $j$ corresponds to the vertex $P_{j}$ then $m_{i, j}=\nu_{i}\left(P_{j}\right)$ for $i=1, \ldots, s$. We build the tree $\theta_{f}(f)$ from the matrix $\mathcal{M}$ by identifying the columns of $\mathcal{M}$ with those non-extremal vertices of $\theta_{f}\left(f_{r}\right)$, for $r=1, \ldots, s$, separately.

We begin by analysing the row $r$. If $a \in\left\{m_{r, j}\right\}_{j=1}^{s\left(f, f_{Y}\right)}$ the set of columns $\mathcal{K}_{a}^{r}:=\left\{j / m_{r, j}=a\right\}$ of $\mathcal{M}$ is non-empty and is clearly in bijection with the set $\mathcal{P}_{a}^{r}=\left\{P\right.$ vertex of $\left.\theta_{f}(f) / \nu_{r}(P)=a\right\}$. Since the set $\mathcal{P}_{a}^{r}$ has a minimum for the valuation $v$, namely the vertex $Q$ of $\theta_{f}\left(f_{r}\right)$ such that $\nu_{r}(P)=a$, it follows by Lemma 17 that there is a unique column $l \in \mathcal{K}_{a}^{r}$, corresponding to $Q$, such that $m_{t, l} \leqslant m_{t, k}$ for $t=1, \ldots, s$ and $k \in \mathcal{K}_{a}^{r}$. This procedure defines a partial order in the columns. We recover the skeleton of the tree $\theta_{f}(f)$ by repeating this procedure for the rows $r=1, \ldots, s$ of $\mathcal{M}$. The vertex of bifurcation of $\theta_{f}\left(f_{r}\right)$ and $\theta_{f}\left(f_{k}\right)$ corresponds to the greatest common column defined by the rows $r$ and $k$. 
To determine the chain $\gamma_{f}$ we use the row $s\left(f, f_{Y}\right)+1$ of $\mathcal{M}$ and the degrees of the irreducible factors of $f$. By Theorem 2 we know that the integer $m_{s\left(f, f_{Y}\right)+1, j}$ is the coefficient of the vertex $P_{j}$, corresponding to the column $j$, in the chain $-\partial \gamma_{f}$. The coefficient of the extremal edge containing the vertex $P_{+\infty}^{(i)}$ in the chain $\gamma_{f}$ is equal to $\operatorname{deg} f_{i}$ for $i=1, \ldots, s$.

Since $\theta_{f}(f)$ is a tree, we recover recursively the coefficients appearing in the segments of the chain $\gamma_{f}$ from the chain $-\partial \gamma_{f}$ and the coefficients $\operatorname{deg} f_{i}$. The chain $\gamma_{f}$ defines the vertices of $\theta_{f_{i}}\left(f_{i}\right)$ and the associated characteristic integers. By using (10) and the $\nu_{i}$ valuation, we recover the valuations $v(P)$ for those non-extremal vertices $P$ of $\theta_{f}\left(f_{i}\right)$ from them.

\section{A geometrical characterization of the bunch decomposition}

We give a geometrical characterization of the bunch decomposition of $f_{Y}$ in terms of the partial embedded resolution $p: \mathcal{Z} \rightarrow \mathbb{C}^{d+1}$ of $f=0$ built by González Pérez in [Gon02, Gon03]. The morphism $p$ is a composition of toric modifications that are canonically determined by the given quasi-ordinary projection, by using the tree $\theta_{f}(f)$. An embedded resolution of $f=0$ is obtained by composing the modification $p$ with any toroidal modification defining a resolution of singularities of $\mathcal{Z}$ (these toroidal modifications always exists, see [KKMS73]).

The exceptional fiber $p^{-1}(0)$ of the modification $p$ is a curve, its irreducible components are complex projective lines. The definition of the modification $p$ induces a bijection $P \mapsto C(P)$ between the non-extremal vertices of the tree $\theta_{f}(f)$ and the irreducible components of the exceptional fiber of $p$.

Theorem 2 establishes a canonical bijection $P \rightarrow b_{P}$ between the non-extremal vertices of the tree $\theta_{f}(f)$ and the bunches of the decomposition of $f_{Y}$ induced by $f$. An irreducible factor $h$ of $f_{Y}$ is a factor of $b_{P}$ if and only if the following equality holds:

$$
\left(\frac{\rho\left(f_{1}, h\right)}{\operatorname{deg} h}, \ldots, \frac{\rho\left(f_{s}, h\right)}{\operatorname{deg} h}\right)=\left(\nu_{1}(P), \ldots, \nu_{s}(P)\right) .
$$

Let $h \in \mathcal{C}_{f}$ be irreducible, we say that $h$ is associated with a non-extremal vertex $P$ of the tree $\theta_{f}(f)$ if the equality $(25)$ holds, or equivalently $\mathcal{N}\left(\psi_{f_{i}}(h)\right)=\operatorname{deg} h / / \nu_{i}(P)$ for $i=1, \ldots, s$; this equivalence is deduced easily by arguing as in the proof of Theorem 1 . The following theorem implies that the $f$-bunch decomposition of $f_{Y}$ is compatible with the bijections above.

Theorem 4. If $h \in \mathcal{C}_{f}$ is associated with a non-extremal vertex $P$ of the tree $\theta_{f}(f)$, then the strict transform of the hypersurface $h=0$ by $p$ only intersects the irreducible component $C(P)$ of $p^{-1}(0)$. The strict transform of $h=0$ does not intersect the strict transform of $f=0$.

We describe the procedure used to build the modification $p$ (for details see [Gon02]). The modification $p$ is a composition $p=\pi_{1} \circ \cdots \circ \pi_{l}$ of toric modifications. The irreducible components of the exceptional fiber $p^{-1}(0)$ are complex projective lines, which can be ordered by $C^{\prime}<C$ if and only if there exists $t>1$ such that the image of $C^{\prime}$ by the modification $p^{\prime}:=\pi_{t} \circ \cdots \circ \pi_{l}$ is a point of $p^{\prime}(C)$ and $p^{\prime}(C)$ is not reduced to a point. The minimal components of $p^{-1}(0)$, with respect to this relation, are the irreducible components of the exceptional fiber $\pi_{1}^{-1}(0)$ of the first toric modification.

The morphism $\pi_{1}$ is the toric modification defined by the dual Newton diagram of $\Sigma(f)$ of the quasi-ordinary polynomial $f \in \mathbb{C}\{X\}[Y]$, when $Y$ is a good coordinate. Such a good coordinate is built by a $\mathbb{C}\{X\}$-automorphism of the polynomial ring $\mathbb{C}\{X\}[Y]$ of the form $Y \mapsto Y+r(X)$. These automorphisms are compatible with the sets $\mathcal{C}_{f}$ and $\mathcal{R} C_{f}$ since they preserve resultants and discriminants of polynomials. We suppose from now on that $Y$ is a good coordinate for $f$, this implies that the Newton polyhedron $\mathcal{N}(f)$ is polygonal and it is completely determined from 


\section{E. R. García Barroso and P. D. González Pérez}

the Eggers-Wall tree $\theta_{f}(f)$. In order to describe this polyhedron we introduce the set $\mathcal{A}_{i}^{(f)}:=$ $\left(M \cap\left\{k\left(f_{i}, f_{j}\right)\right\}_{j}\right) \cup\left\{\lambda_{1}^{(i)}\right\}$ for $1 \leqslant i \leqslant s$. By Lemma 1 , if the set $\mathcal{A}_{i}^{(f)}$ is non-empty it is totally ordered. We define $\lambda_{\kappa(i)}^{(f)}$ by

$$
\lambda_{\kappa(i)}^{(f)}:=\left\{\begin{array}{ll}
\min \mathcal{A}_{i}^{(f)} & \text { if } \mathcal{A}_{i}^{(f)} \neq \emptyset, \\
+\infty & \text { otherwise, }
\end{array} \quad \text { for } i=1, \ldots, s .\right.
$$

The polyhedron $\mathcal{N}\left(f_{i}\right)$ has only one compact edge of inclination equal to $\lambda_{\kappa(i)}^{(f)}$ if $\lambda_{\kappa(i)}^{(f)} \neq+\infty$. The case $\lambda_{\kappa(i)}^{(f)}=+\infty$ may only happen for one index $i$ and in that case $f_{i}$ is a good coordinate for $f$, i.e. in this case we change of coordinates in order to have $Y=f_{i}$. We need the following lemma (see [Gon02, lemma 3.15]).

LEMma 28. If the term $X^{\lambda}$ appears in the expansions of the roots of $f_{j}, \lambda \leqslant \lambda_{\kappa(i)}^{(f)}$ and $\lambda \neq \lambda_{\kappa(i)}^{(f)}$, then we have that $\lambda \geqslant k\left(f_{i}, f_{j}\right)$ and the equality $\lambda=k\left(f_{i}, f_{j}\right)$ implies that $k\left(f_{i}, f_{j}\right)=\lambda_{1}^{(j)}$. The set $\left\{\lambda_{\kappa(1)}^{(f)}, \ldots, \lambda_{\kappa(s)}^{(f)}\right\}$ is totally ordered by (2) and its intersection with the reference lattice $M$ is defined by its maximal element or empty.

The exceptional fiber of the toric modification $\pi_{1}$ is described by (19). We denote by

$$
C\left(P_{\lambda_{\kappa(i)}^{(i)}}^{(f)}\right)
$$

the irreducible component of $\pi_{1}^{-1}(0)$, which is equal to the closure of the orbit associated with the cone of $\Sigma(f)$, orthogonal to the compact edge $\mathcal{E}_{i}$ of $\mathcal{N}(f)$ with inclination $\lambda_{\kappa(i)}^{(f)} \neq+\infty$.

The following lemma describes some properties of the strict transform of germs defined by the irreducible factors $f_{k}$ of the quasi-ordinary polynomial $f$, by $\pi_{1}$ (see [Gon02, Proposition 3.32]).

LEMma 29. The strict transform of $f_{i}=0$ by $\pi_{1}$ is a germ at the point of intersection $o_{1}^{(i)}$ with $\pi_{1}^{-1}(0)$. The point $o_{1}^{(i)}$ belongs to only one irreducible component of $\pi_{1}^{-1}(0)$ equal to

$$
\begin{cases}C\left(P_{\left.\max \left\{\lambda_{\kappa(j)}^{(f)}\right\}_{j=1, \ldots, s}^{j \neq i}\right)}^{(*)}\right) & \text { if } \lambda_{\kappa(i)}^{(f)}=+\infty \\ C\left(P_{\lambda_{\kappa(i)}^{(f)}}^{(i)}\right) & \text { otherwise. }\end{cases}
$$

If $\lambda_{\kappa(i)}^{(f)} \neq+\infty$, the point $o_{1}^{(i)}$ is determined as scheme by the root $c_{f_{i}} \in \mathbb{C}^{*}$ of $p\left(f_{i}, \mathcal{E}_{i}\right)$, counted with multiplicity (where $p\left(f_{i}, \mathcal{E}_{i}\right)$ is the polynomial in one variable defined from the symbolic restriction of $f$ to the compact edge $\mathcal{E}_{i}$ of $\mathcal{N}\left(f_{i}\right)$ by (20)). We have that

$$
C\left(P_{\lambda_{\kappa(i)}^{(f)}}^{(i)}\right)=C\left(P_{\lambda_{\kappa(j)}^{(j)}}^{(j)}\right) \Leftrightarrow k\left(f_{i}, f_{j}\right) \geqslant \lambda_{\kappa(i)}^{(f)}=\lambda_{\kappa(j)}^{(f)}
$$

and

$$
o_{1}^{(i)}=o_{1}^{(j)} \Leftrightarrow c_{f_{i}}=c_{f_{j}} \Leftrightarrow k\left(f_{i}, f_{j}\right)>\lambda_{\kappa(i)}^{(f)} .
$$

The key inductive step in the embedded resolution procedure is that the strict transform of $f_{i}=0$ at the point $o_{1}^{(i)}$ is a toric quasi-ordinary singularity, ${ }^{3}$ with a canonical 'quasi-ordinary' projection. These singularities are hypersurfaces of affine toric varieties, for instance the strict transform of $f=0$ at the point $o_{1}^{(i)}$ is defined by the vanishing of a monic polynomial $f^{\prime}$ in one variable with coefficients in the ring $\mathbb{C}\left\{\Delta_{d}^{\vee} \cap\left(M+\lambda_{\kappa(i)}^{(f)} \mathbb{Z}\right)\right\}$. The polynomial $f^{\prime}$ is quasi-ordinary: its discriminant

\footnotetext{
${ }^{3}$ This means that there is a finite projection onto a germ of affine toric variety which is unramified outside its torus (see [Gon02, Gon00a]).
} 


\section{DECOMPOSITION IN BUNCHES OF THE CRITICAL LOCUS OF A QUASI-ORDINARY MAP}

is the product of a monomial by a unit of this ring. The definition of characteristic monomials and the Eggers-Wall tree introduced in the first section generalize to this setting, in the form given in Lemma 23 (see [Gon02]). In particular the Eggers-Wall tree of $f^{\prime}$ is determined from $\theta_{f}(f)$ by the following proposition (see [Gon02, Proposition 3.22]).

Proposition 30. If $\lambda_{\kappa(i)}^{(f)} \neq+\infty$, the Eggers-Wall tree $\theta_{f^{\prime}}\left(f^{\prime}\right)$ associated with the strict transform of $f$ at the point $o_{1}^{(i)}$ is obtained from $\theta_{f}(f)$ by removing the segment

$$
\left[P_{0}^{(j)}, P_{\lambda_{\kappa(i)}^{(f)}}^{(j)}[\right.
$$

from the sub-tree of $\theta_{f}(f)$ given by $\bigcup \theta_{f}\left(f_{j}\right)$, for those irreducible factors $f_{j}$ with order of coincidence $>\lambda_{\kappa(i)}^{(f)}$ with $f_{i}$. The new lattice valuation is

$$
\tilde{v}^{\prime}(P)=\tilde{v}(P)-\tilde{v}\left(P_{\lambda_{\kappa(i)}^{(f)}}^{\left(f_{i}\right)}\right)
$$

The coefficients of the 1-chain $\gamma_{f^{\prime}}$ are obtained from those of $\gamma_{f}$ by dividing by the index of $\lambda_{\kappa(i)}^{(f)}$ over the old reference lattice $M$. The new reference lattice cone is $\left(\Delta_{d}^{\vee}, M+\lambda_{\kappa(i)}^{(f)} \mathbb{Z}\right)$.

Proposition 30 allows us to extend the natural bijection between the components of the exceptional fiber of $\pi_{1}$ and the subset

$$
\left\{P_{\lambda_{\kappa(1)}^{(f)}}^{(1)}, \ldots, P_{\lambda_{\kappa(s)}^{(f)}}^{(s)}\right\}
$$

of vertices of $\theta_{f}(f)$, inductively between the components of $p^{-1}(0)$ and the set of non-extremal vertices of $\theta_{f}(f)$.

Lemma 31. Let $h \in \mathcal{C}_{f}$ be an irreducible polynomial associated with a non-extremal vertex $P$ of $\theta_{f}(f)$. Let

$$
C\left(P_{\lambda_{\kappa(i)}^{(i)}}^{(f)}\right)
$$

be the minimal component of $p^{-1}(0)$ that is $\leqslant C(P)$. Then we have that $\lambda_{\kappa(i)}^{(f)} \neq+\infty$, and the point of intersection $o_{1}^{(h)}$ of the strict transform of $h=0$ by $\pi_{1}$ with the fiber $\pi_{1}^{-1}(0)$ only belongs to the irreducible component

$$
C\left(P_{\lambda_{\kappa(i)}^{(i)}}^{(f)}\right)
$$

of $\pi_{1}^{-1}(0)$. Moreover, we have that $\mathcal{N}(h) \subset \operatorname{deg} h / / \lambda_{\kappa(i)}^{(f)}$ and the following assertions hold.

(i) The polynomial $p(h, \mathcal{E}) \in \mathbb{C}[t]$ obtained from the symbolic restriction of $h$ to the compact edge $\mathcal{E}$ of the polyhedron $\operatorname{deg} h / / \lambda_{\kappa(i)}^{(f)}$ by (20) has only one complex root $c_{h}$ (counted without multiplicity). We have that

$$
\left.o_{1}^{(h)}=o_{1}^{(i)} \Leftrightarrow c_{h}=c_{f_{i}} \Leftrightarrow v(P)>\lambda_{\kappa(i)}^{(f)} \quad \text { (respectively } o_{1}^{(h)} \neq o_{1}^{(i)} \Leftrightarrow c_{h} \neq c_{f_{i}} \Leftrightarrow v(P)=\lambda_{\kappa(i)}^{(f)}\right) .
$$

(ii) The case $\mathcal{N}(h) \neq \operatorname{deg} h / / \lambda_{\kappa(i)}^{(f)}$ happens if and only if $c_{h}=0$, and then $v(P)=\max \left\{\lambda_{\kappa(j)}^{(f)}\right\}_{j=1}^{s}$.

Proof. We first deal with the case $h \in \mathcal{R} C_{f}$. We consider $h$ as an irreducible factor of the quasiordinary polynomial $f h$. The hypothesis means that the vertex $P=P_{k(f, h)}^{h}$ of $\theta_{f h}(f)$ belongs to $\theta_{f}(f)$, therefore $\theta_{f h}(f)=\theta_{f}(f)$. There exists $1 \leqslant i \leqslant s$ such that $P \in \theta_{f}\left(f_{i}\right)$ and $v(P)=$ $\max _{l=1, \ldots, s}\left\{k\left(f_{l}, h\right)\right\}=k\left(f_{i}, h\right)$ is the exponent of a term appearing with non-zero coefficient in the expansion of the roots of $f_{i}$. 


\section{E. R. García Barroso and P. D. González Pérez}

The minimal component $p^{-1}(0)$ that is $\leqslant C_{P}$, is the unique component of $\pi_{1}^{-1}(0)$ that is $\leqslant C_{P}$. We show that this component is

$$
C\left(P_{\lambda_{\kappa(i)}^{(i)}}^{(i)}\right)
$$

It follows from the hypothesis and the definitions that $\lambda_{\kappa(l)}^{(f h)}=\lambda_{\kappa(l)}^{(f)}$, for $l=1, \ldots, s$. Since the exponent $v(P)$ appears on a term of the expansions of the roots of $f_{i}$ with non-zero coefficient, we have that $\lambda_{\kappa(i)}^{(f)} \leqslant v(P)$, in the totally ordered set $V_{f h}\left(f_{i}\right)=V_{f}\left(f_{i}\right)$. If $\lambda_{\kappa(i)}^{(f)}<v(P)$ the assertion follows by Lemma 29, applied to $f_{i}$ and $h$ viewed as irreducible factors of the quasi-ordinary polynomial $f h$. If $\lambda_{\kappa(i)}^{(f)}=v(P)$, we have that the exponents appearing in the expansions of the roots of $f_{i}$ with non-zero coefficient are $\geqslant \lambda_{\kappa(i)}^{(f)}$, since $Y$ is a good coordinate for $f$. The equality $v(P)=k\left(f_{i}, h\right)=\lambda_{\kappa(i)}^{(f h)}$ implies that the previous statement is also true for the expansions of the roots of $h$, therefore we have the inclusion $\mathcal{N}(h) \subset \operatorname{deg} h / / \lambda_{\kappa(i)}^{(f)}$. These polyhedra are equal if and only if $p(h, \mathcal{E})$ has a non-zero complex root $c_{h}$. We prove that this is always the case when there exists $1 \leqslant j \leqslant s$ such that $\lambda_{\kappa(i)}^{(f)}<\lambda_{\kappa(j)}^{(f)}$ (eventually $\left.\lambda_{\kappa(j)}^{(f)}=+\infty\right)$.

By hypothesis, we have that

$$
\lambda_{\kappa(l)}^{(f)}=\lambda_{\kappa(l)}^{(f h)} \quad \text { for } 1 \leqslant l \leqslant s \quad \text { and } \quad k\left(f_{j}, h\right) \leqslant k\left(f_{i}, h\right)=\lambda_{\kappa(i)}^{(f h)}<\lambda_{\kappa(j)}^{(f h)} .
$$

It follows from Lemma 28 applied to $f h$ that $k\left(f_{j}, h\right)=\lambda_{1}^{(h)}$ and that $k\left(f_{j}, f_{i}\right)=\lambda_{1}^{(i)}=\lambda_{\kappa(i)}^{(f h)}$. Then the inequality $\lambda_{1}^{(h)} \leqslant k\left(f_{i}, h\right)=\lambda_{\kappa(i)}^{(f h)}$ implies that $\lambda_{1}^{(h)}=\lambda_{\kappa(i)}^{(f h)}$ by definition of the order of coincidence. We deduce that $c_{h} \neq 0$ and $\mathcal{N}(h)=\operatorname{deg} h / / \lambda_{\kappa(i)}^{(f)}$.

If these polyhedra are not equal, then the intersection of the compact face $\mathcal{E}$ of $\operatorname{deg} h / / \lambda_{\kappa(i)}^{(f)}$ with $\mathcal{N}(h)$ is reduced to the point $(0, \operatorname{deg} h)$, and therefore $c_{h}=0$.

The rest of the assertion follows from Lemma 29 applied to the quasi-ordinary polynomial $f h$.

We prove that this result also holds for $h \in \mathcal{C}_{f}$ by using toric base changes of $\S 6$. Let $\tau \subset \Delta_{d}$ be any regular cone of dimension $d$ such that $h^{(\tau)} \in \mathcal{R} C_{f(\tau)}$. The polynomial $p(h, \mathcal{E})$ coincides with $p\left(h^{(\tau)}, \mathcal{E}\right)$ by Lemmas 24 and 26. It follows from these lemmas that $\mathcal{N}(h) \subset \operatorname{deg} h / / \lambda_{\kappa(i)}^{(f)}$, with equality if $c_{h} \neq 0$ and that if $c_{h}=0$, the intersection of the compact face $\mathcal{E}$ of $\operatorname{deg} h / / \lambda_{\kappa(i)}^{(f)}$ with $\mathcal{N}(h)$ is reduced to the point $(0, \operatorname{deg} h)$. The geometric assertion follows from Lemma 15 if $c_{h} \neq 0$, and from Lemma 16, if $c_{h}=0$.

Proof of Theorem 4. If

$$
P=P_{\lambda_{\kappa(i)}^{(i)}}^{(f)}
$$

for some $i$, we deduce from Lemma 31 that the strict transform $\mathcal{H}^{\prime}$ of $h=0$ by $\pi_{1}$ only meets the component $C(P)$ of $\pi_{1}^{-1}(0)$ and it does not intersect the strict transforms of $f=0$. This implies that a neighborhood of $\mathcal{H}^{\prime}$ will not be modified by the toric modifications $\pi_{2}, \ldots, \pi_{l}$ and proves the theorem in this case.

If

$$
C\left(P_{\lambda_{\kappa(i)}^{(f)}}^{(i)}\right)<C(P)
$$

we apply Lemma 31. We consider the polynomial $h^{\prime}$ defining the strict transform of $h$ (which is a germ at the point $\left.o_{1}^{(i)}\right)$. It follows from Proposition 30 and another application of Lemmas 24 and 26 that $h^{\prime}$ is associated with $P$ viewed on the tree $\theta_{f^{\prime}}\left(f^{\prime}\right)$ (where $f^{\prime}$ denotes the quasi-ordinary polynomial defining the strict transform of $f$ by $\pi_{1}$ at the point $\left.o_{1}^{(i)}\right)$. If $C(P)$ is minimal between those components of $p^{-1}(0)$ corresponding to the non-extremal vertices of $\theta_{f^{\prime}}\left(f^{\prime}\right)$ we can apply the arguments of the previous case, otherwise we obtain the result by iterating the procedure. 


\section{DECOMPOSITION IN BUNCHES OF THE CRITICAL LOCUS OF A QUASI-ORDINARY MAP}

\subsection{A theorem of Lê et al. revisited}

If $d=1$, then $f \in \mathbb{C}\{X\}[Y]$ defines the germ of a complex analytic plane curve in $\left(\mathbb{C}^{2}, 0\right)$. The minimal embedded resolution of $(S, 0)$ is the modification $\Pi: \mathcal{X} \rightarrow \mathbb{C}^{2}$ defined by the composition of the minimal sequence of points blow-ups, such that the total transform of $f=0$ is a normal crossing divisor. The dual graph $\mathcal{G}(\Pi, 0)$ (respectively the total dual graph $\mathcal{G}(\Pi, f)$ ) is the graph obtained from the exceptional divisor $\Pi^{-1}(0)$ (respectively from the total transform $\Pi^{-1}(\{f=0\})$ ) by associating a vertex to any irreducible component and joining with a segment those vertices whose associated components have non-empty intersection. The valency of a vertex $P$ of a finite graph is the number of edges of the graph that contain the vertex $P$. We denote by $\# 1$ the component of $\mathcal{G}(\Pi, f)$ which corresponds to the first blow-up. We define for a vertex $P$ of $\mathcal{G}(\Pi, f)$ the integer

$$
\omega(P):= \begin{cases}\text { valency of } P \text { in } \mathcal{G}(\Pi, f) & \text { if } P \neq \# 1 \\ 1+\text { valency of } P \text { in } \mathcal{G}(\Pi, f) & \text { if } P=\# 1 .\end{cases}
$$

A vertex $P$ of $\mathcal{G}(\Pi, f)$ with $\omega(P)=1$ (respectively with $\omega(P) \geqslant 3$ ) is called an extremal vertex (respectively a rupture vertex). A dead arc of the graph $\mathcal{G}(\Pi, f)$ is a polygonal path in $\mathcal{G}(\Pi, 0)$ joining a extremal vertex to any rupture vertex of $\mathcal{G}(\Pi, f)$, which does not contain any other rupture vertex. The dual graph $\mathcal{G}(\Pi, f)$ defines a natural stratification of $\Pi^{-1}(\{f=0\})$, the zero-dimensional strata are in bijection with the segments of $\mathcal{G}(\Pi, f)$, each segment corresponds to the intersection of the irreducible components associated with its vertices. The one-dimensional strata are in bijection with the vertices, the stratum corresponding to a vertex of $\mathcal{G}(\Pi, f)$ is the set of points of the corresponding component that do not belong to any other component of $\Pi^{-1}(\{f=0\})$. By this correspondence a subset of $\Pi^{-1}(\{f=0\})$ is associated with any subgraph of $\mathcal{G}(\Pi, f)$.

With this notation, the main result of Lê et al. in [LMW89] is as follows.

Theorem 5. Denote by $\mathcal{Q}(\Pi, f)$ the set associated with the subgraph of $\mathcal{G}(\Pi, f)$ defined by the rupture vertices and dead arcs. If $X=0$ is not contained in the tangent cone of the plane curve germ $f(X, Y)=0$ the intersection of the exceptional divisor $\Pi^{-1}(0)$ with the strict transform of the polar curve $f_{Y}=0$ is contained $\mathcal{Q}(\Pi, f)$ and meets any connected component of $\mathcal{Q}(\Pi, f)$.

Proof. If $X=0$ is not contained in the tangent cone of the plane curve germ $f=0$ the minimal embedded resolution $\Pi$ is the composition of the partial embedded resolution $p: \mathcal{Z} \rightarrow \mathbb{C}^{2}$ used in the previous section, with a finite number of local toric modifications at the isolated singular points of the normal variety $\mathcal{Z}$ (see [Gon02, § 3.3.4] for details). The notions of dual graph $\mathcal{G}(p, 0)$ and total dual graph $\mathcal{G}(p, f)$ can be defined in an analogous way for $p$. In particular, we have that $\mathcal{G}(p, 0)$ is combinatorially isomorphic to the tree $\theta_{f}(f)$ minus its extremal segments and that there is a natural inclusion of the vertices of $\mathcal{G}(p, 0)$ in the vertices of $\mathcal{G}(\Pi, 0)$ whose image is the subset of rupture vertices of $\mathcal{G}(\Pi, f)$. The dual graph $\mathcal{G}(p, f)$ is associated in an analogous manner with a natural stratification of $p^{-1}(\{f=0\})$, in such a way that a subset of $p^{-1}(\{f=0\})$ corresponds to any subgraph of $\mathcal{G}(p, f)$. We denote by $\mathcal{Q}(p, f)$ the subset of $p^{-1}(\{f=0\})$ corresponding to the set of vertices of $\mathcal{G}(p, f)$.

An irreducible factor $h$ of $f_{Y}$ is associated with a non-extremal vertex $P$ of $\theta_{f}(f)$ by Theorem 2 . The strict transform of $h$ by $p$ intersects $p^{-1}(0)$ at a point $o_{h}$, which belongs to only one irreducible component of $p^{-1}(0)$, namely the component $C(P)$ associated with $P$, and does not belong to the strict transform of $f$, by Theorem 4 . In other terms the point $o_{h}$ belongs to $\mathcal{Q}(p, f)$. If $\Pi=p^{\prime} \circ p$, then we have that $\mathcal{Q}(\Pi, f)=\left(p^{\prime}\right)^{-1}(\mathcal{Q}(p, f))$ and the result follows.

Remark 32. Suppose that the component corresponding to $C(P)$ in $\Pi^{-1}(0)$ belongs to a dead arc. If $C(P)$ is minimal for $p^{-1}(0)$ then $v(P)=\max \left\{\lambda_{\kappa(j)}^{(f)}\right\}$, which is necessarily less than $+\infty$. If $C(P)$ is not minimal, after some toric modifications, $C(P)$ is minimal for the strict transform of $f$ and an analogous result holds. 


\section{E. R. García Barroso and P. D. González Pérez}

By definition there is no dead arc corresponding to $\min \left\{\lambda_{\kappa(j)}^{(f)}\right\}$ since the extremal point \#1 is not considered in the definition of dead arcs.

\section{The case of Laurent quasi-ordinary polynomials}

The class of Laurent quasi-ordinary polynomials was introduced by Popescu-Pampu in [Pop01] (see also [Pop04]), by analogy with the case of meromorphic plane curves studied by Abhyankar and Assi (see [AA99]). Popescu-Pampu proved a decomposition theorem for the derivative of any polynomial in the class such that the derivative itself is also Laurent quasi-ordinary. In this section we generalize this result to any Laurent quasi-ordinary polynomial by translating in an equivalent manner, the properties of the bunch decomposition of the derivative of a quasi-ordinary polynomial from the Laurent case to holomorphic case (characterized in Theorem 2). This reduction is partially inspired by an argument of Kuo and Parusinski comparing the plane curve meromorphic case with the holomorphic case (see [KP04]).

We denote by $\mathbb{C}\langle X\rangle$ the ring of Laurent power series in $X=\left(X_{1}, \ldots, X_{d}\right)$, i.e. the ring of fractions $\mathbb{C}\{X\}\left[X_{1}^{-1}, \ldots, X_{d}^{-1}\right]$. A Laurent polynomial $F \in \mathbb{C}\langle X\rangle[Y]$ admits a Newton polyhedron. The definition does not coincide with that used in the holomorphic case. If $F=\sum c_{\alpha, i} X^{\alpha} Y^{i}$, its Newton polyhedron is the convex hull of the set $\bigcup_{c_{\alpha, i} \neq 0}(\alpha, i)+\left(\mathbb{R}_{\geqslant 0}^{d} \times\{0\}\right)$. A Laurent monic polynomial $F \in \mathbb{C}\langle X\rangle[Y]$ is quasi-ordinary if the discriminant $\Delta_{Y}(f)$ is of the form $\Delta_{Y}(f)=X^{\delta} \epsilon$ where $\delta \in \mathbb{Z}^{d}$ and $\epsilon$ is a unit in the ring of power series $\mathbb{C}\{X\}$. We extend the definition of polynomials comparable with a quasi-ordinary polynomial to the Laurent case. In particular if $F$ is a Laurent quasi-ordinary polynomial we define the type of $F_{Y}$ as in the holomorphic case (see Definition 4). We relate Laurent monic polynomials with monic holomorphic polynomials by the following lemma.

Lemma 33. Let $F=F(Y)$ be a monic polynomial with coefficients in $\mathbb{C}\langle X\rangle$. Then there exists a vector $q \in \mathbb{Z}^{d}$ such that the monic polynomial $f$ defined by

$$
f:=X^{-\operatorname{deg}(F) q} F\left(X^{q} Y\right)
$$

belongs to $\mathbb{C}\{X\}[Y]$. In this case we have:

(i) if $F$ is quasi-ordinary the same holds for $f$;

(ii) if $F=F_{1} \cdots F_{s}$ is the factorization in irreducible monic polynomials the same holds for $f=$ $f_{1} \cdots f_{s}$ where the polynomials $f_{i}$ are defined from $F_{i}$ by (28);

(iii) the polynomial $r$ defined from $R=F_{Y}$ by (28) is equal to $r=f_{Y}$.

Proof. The polyhedron $\mathcal{N}(F) \subset \mathbb{R}^{d} \times \mathbb{R}$ is contained in an affine cone $W$ of the form

$$
W=[(0, \operatorname{deg} F),(a, 0)]+\left(\mathbb{R}^{d} \times\{0\}\right)
$$

for some integral vector $a \in(\operatorname{deg} F) \mathbb{Z}^{d}$. If $q:=(1 / \operatorname{deg} F) a$, then we obtain that the polynomial $f$ defined by (28) belongs to $\mathbb{C}\{X\}[Y]$. The idea is that the one-dimensional face $[(0, \operatorname{deg} F),(a, 0)]$ of the cone $W$ corresponds to the segment $[(0, \operatorname{deg} f),(0,0)]$ in the Newton polyhedron of $f$. If $F=\sum_{i=0}^{n} a_{i} Y^{i}$, then $f=X^{-\operatorname{deg}(F) q}\left(\sum_{i=0}^{n} a_{i} X^{i q} Y^{i}\right)$, and if $R=\partial F / \partial Y$, then we obtain that the polynomial

$$
\frac{\partial f}{\partial Y}=X^{(-\operatorname{deg}(F)+1) q}\left(\sum_{i=1}^{n} i a_{i} X^{(i-1) q} Y^{i-1}\right)
$$

is equal to $r$. It follows from quasi-homogeneity and homogeneity properties of the generic discriminant and resultant (see [GKZ94, pp. 398-399]) that $f$ is quasi-ordinary if $F$ is. See also the proof of Theorem 3 of [Gon00a] for details. 


\section{DECOMPOSITION IN BUNCHES OF THE CRITICAL LOCUS OF A QUASI-ORDINARY MAP}

It remains to prove assertion (ii). Recall that if a domain $A$ is integrally closed with fraction field $K$, then the factorization of a monic polynomial in $A[Y]$ as product of monic irreducible factors coincides over $A[Y]$ and $K[Y]$. It is easy to see that the rings $\mathbb{C}\{X\} \subset \mathbb{C}\langle X\rangle$ are integrally closed and have the same fraction field $L$. For any fixed $q \in \mathbb{Z}^{d}$ the multiplicative endomorphism of $\mathbb{C}\langle X\rangle[Y] \backslash\{0\}$ defined by (28) is an automorphism which preserves degrees and monic polynomials, and extends to a multiplicative automorphism of $L[Y] \backslash\{0\}$. Therefore, the factorization in monic irreducible factors of a monic polynomial $F \in \mathbb{C}\langle X\rangle[Y]$ corresponds by this mapping to the factorization in monic irreducible factors of $f$ in $L[Y]$; if, in addition, $f$ belongs to $\mathbb{C}\{X\}[Y]$, then this factorization holds over $\mathbb{C}\{X\}[Y]$ since $\mathbb{C}\{X\}$ is integrally closed.

Let $F$ be a Laurent quasi-ordinary polynomial. Let $q$ be a vector in $\mathbb{Z}^{d}$ verifying the assertion of Lemma 33. If $F_{Y}=H_{1} \cdots H_{r}$ is the factorization in monic irreducible polynomials, then the same holds for $f_{Y}=h_{1} \ldots h_{r}$ by Lemma 33 (where the polynomials $h_{i}$ are defined from $H_{i}$ by (28)).

We obtain, by using the quasi-homogeneity and homogeneity properties of the generic resultant (see [GKZ94, pp. 398-399]), that for any $i, j$

$$
\operatorname{Res}_{Y}\left(f_{j}, h_{i}\right)=X^{-\operatorname{deg}\left(H_{i}\right) \operatorname{deg}\left(F_{j}\right) q} \operatorname{Res}_{Y}\left(F_{j}, H_{i}\right)
$$

and since $\operatorname{deg} H_{i}=\operatorname{deg} h_{i}$ and $\operatorname{deg} F_{i}=\operatorname{deg} f_{i}$ for all $i$, we obtain that

$$
\frac{\rho\left(F_{j}, H_{i}\right)}{\operatorname{deg} H_{i}}=\frac{\rho\left(f_{j}, h_{i}\right)}{\operatorname{deg} h_{i}}+\operatorname{deg}\left(f_{j}\right) q
$$

We have proved the following result.

Proposition 34. The bunches of the F-decomposition of $F_{Y}$ correspond to the bunches of the $f$-decomposition of $f_{Y}$ by the transformation (28). The type of $F_{Y}$ is obtained from the type of $f_{Y}$ by (29).

\section{An example}

The polynomial $f_{i, j}:=\left(Y^{2}-i X_{1}^{3} X_{2}^{2}\right)^{2}-j X_{1}^{5} X_{2}^{4} Y$ is quasi-ordinary with characteristic exponents $\lambda_{1}=\left(\frac{3}{2}, 1\right)$ and $\lambda_{2}=\left(\frac{7}{4}, \frac{3}{2}\right)$, and integers $n_{1}=n_{2}=2$ for any $i, j \in \mathbb{C}^{*}$. The equation is obtained by defining a deformation of the monomial variety associated with a quasi-ordinary hypersurface (see [Gon02]) in an analogous manner as the deformation of the monomial curve associated with a plane branch studied in [Tei86].

The Eggers-Wall tree associated with the polynomial $f=f_{1,1} f_{1,2} f_{2,1} f_{2,2}$ is indicated in Figure 1 . We have that $v\left(P_{1}\right)=\lambda_{1}$ and $v\left(P_{2}\right)=v\left(P_{3}\right)=\lambda_{2}$. The edges are labeled with the coefficients of the chain $\gamma_{f}$ thus we have that $-\partial \gamma_{f}=4 P_{1}+6 P_{2}+6 P_{3}$.

\begin{tabular}{|c|c|c|c|}
\hline$f_{1,1}$ & $(6,4)$ & $\left(\frac{13}{2}, 5\right)$ & $(6,4)$ \\
\hline$f_{1,2}$ & $(6,4)$ & $\left(\frac{13}{2}, 5\right)$ & $(6,4)$ \\
\hline$f_{2,1}$ & $(6,4)$ & $(6,4)$ & $\left(\frac{13}{2}, 5\right)$ \\
\hline \multirow[t]{4}{*}{$f_{2,2}$} & $(6,4)$ & $(6,4)$ & $\left(\frac{13}{2}, 5\right)$ \\
\hline & 3 & 6 & 6 \\
\hline & - & - & - \\
\hline & $P_{1}$ & $P_{2}$ & $P_{3}$ \\
\hline
\end{tabular}

We determine the type of $f_{Y}$ by using Proposition 5 and Theorem 2:

We compute the Newton polyhedra of the polynomials $\psi_{f_{1,1}}\left(f_{Y}\right)$ and $\psi_{f}\left(f_{Y}\right)$ by using Theorem 1 . We obtain that

$$
\mathcal{N}\left(\psi_{f_{i, j}}\left(f_{Y}\right)\right)=\frac{3}{\overline{(6,4)}}+\frac{6}{\overline{(6,4)}}+\frac{6}{\overline{(13 / 2,5)}}=\frac{9}{\overline{(6,4)}}+\frac{6}{\overline{(13 / 2,5)}} \quad \text { for } i, j \in\{1,2\}
$$


E. R. García Barroso and P. D. González PÉrez

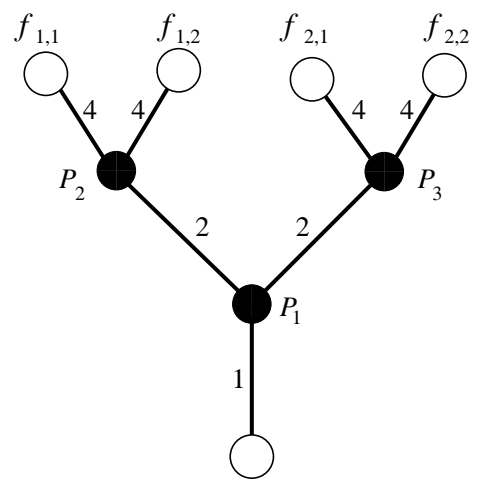

Figure 1. The Eggers-Wall tree associated with $f$.

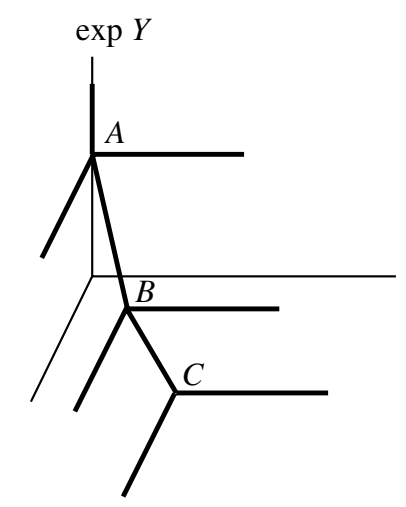

Figure 2. A polygonal polyhedron.

and

$$
\mathcal{N}\left(\psi_{f}\left(f_{Y}\right)\right)=\frac{3}{\overline{(24,16)}}+\frac{6}{\overline{(25,18)}}+\frac{6}{\overline{(25,18)}}=\frac{3}{\overline{(24,16)}}+\frac{12}{\overline{(25,18)}}
$$

It follows that the polyhedra $\mathcal{N}\left(\psi_{f_{i, j}}\left(f_{Y}\right)\right)$ coincide in this example for $i, j \in\{1,2\}$. In particular, the example shows that the only datum of these polyhedra does not allow us to distinguish between the different irreducible factors of $f$. The polyhedron obtained for $\mathcal{N}\left(\psi_{f_{i, j}}\left(f_{Y}\right)\right.$ ) (respectively for $\left.\mathcal{N}\left(\psi_{f}\left(f_{Y}\right)\right)\right)$ is of the form of given in Figure 2 where the vertices are $A=((0,0), 15)$, $B=((54,36), 6)$ and $C=((93,66), 0)$ (respectively $A=((0,0), 15), B=((72,48), 12)$ and $C=((372,264), 0))$.

If we are given the type of $f$ we recover the skeleton of the tree by noting that $\theta_{f}\left(f_{i, j}\right)$ has two non-extremal vertices, corresponding to the different values appearing on the associated row. The corresponding minimal columns for $i=j=1$ (and for $i=1, j=2$ ) are $P_{1}$ and $P_{2}$ with $P_{1}<P_{2}$. Thus the column $P_{2}$ corresponds to the point of bifurcation of $\theta_{f}\left(f_{1,1}\right)$ and $\theta_{f}\left(f_{1,2}\right)$. The columns corresponding to the non-extremal vertices of $\theta_{f}\left(f_{i, j}\right)$ for $i=2, j=1,2$ are $P_{1}<P_{3}$, the bigger column corresponds with the point of bifurcation of $\theta_{f}\left(f_{2,1}\right)$ and $\theta_{f}\left(f_{2,2}\right)$ and the first is the point of bifurcation of $\theta_{f}\left(f_{1,1}\right)$ and $\theta_{f}\left(f_{2,1}\right)$. The coefficient of the edge $\overline{P_{1} P_{2}}$ on $\gamma_{f}$ is equal to $\operatorname{deg} f_{1,1}+\operatorname{deg} f_{1,2}-c_{P_{2}}=4+4-6=2$ and we obtain the same value for $\overline{P_{1} P_{3}}$. Then we recover the characteristic exponents $v\left(P_{i}\right)$ by using Proposition 5 . 


\section{DECOMPOSITION IN BUNCHES OF THE CRITICAL LOCUS OF A QUASI-ORDINARY MAP}

\section{ACKNOWLEDGEMEnTS}

We are grateful to Bernard Teissier for his suggestions and comments.

\section{REFERENCES}

AA99 S. S. Abhyankar and A. Assi, Jacobian of meromorphic curves, Proc. Indian Acad. Sci. Math. Sci. 109 (1999), 117-163.

Abh55 S. S. Abhyankar, On the ramification of algebraic functions, Amer. J. Math. 77 (1955), 575-592.

BS92 L. J. Billera and B. Sturmfels, Fiber polytopes, Ann. of Math. (2) 135 (1992), 527-549.

Egg82 H. Eggers, Polarinvarianten und die Topologie von Kurvensingularitäten, Bonner Mathematische Schriften, vol. 147 (Universität Bonn, Mathmatische Institut, 1982).

Ewa96 G. Ewald, Combinatorial convexity and algebraic geometry (Springer, Berlin, 1996).

Gar00 E. R. García Barroso, Sur les courbes polaires d'une courbe plane réduite, Proc. London Math. Soc. (3) 81 (2000), 1-28.

Gau88 Y.-N. Gau, Embedded topological classification of quasi-ordinary singularities, Mem. Amer. Math. Soc. 74 (388) (1988), 109-129.

GKZ94 I. M. Gel'fand, M. M. Kapranov and A. V. Zelevinsky, Discriminants, resultants and multidimensional determinants (Birkhäuser, Boston, MA, 1994).

GMN03 P. D. González Pérez, L. J. McEwan and A. Némethi, The zeta function of a quasi-ordinary singularity II, Contemp. Math., vol. 324 (American Mathematical Society, Providence, RI, 2003), $109-122$.

Gon00a P. D. González Pérez, Singularités quasi-ordinaires toriques et polyèdre de Newton du discriminant, Canad. J. Math. 52 (2000), 348-368.

Gon00b P. D. González Pérez, Quasi-ordinary singularities via toric geometry, Tesis Doctoral, Universidad de La Laguna (2000).

Gon02 P. D. González Pérez, Étude des singularités quasi-ordinaires d'hypersurfaces au moyen de la géometrie torique, Thèse de doctorat, Université de Paris 7 (2002).

Gon03 P. D. González Pérez, Toric embedded resolutions of quasi-ordinary hypersurface singularities, Ann. Inst. Fourier (Grenoble) $\mathbf{5 3}$ (2003), 1819-1881.

GT99 E. R. García Barroso and B. Teissier, Concentration multi-échelles de courbure dans des fibres de Milnor, Comment. Math. Helv. 74 (1999), 398-418.

Jun08 H. W. E. Jung, Darstellung der Funktionen eines algebraischen Körpers zweier unabhängigen Veränderlichen $x, y$ in der Umgebung einer stelle $x=a, y=b$, J. reine angew. Math. 133 (1908), 289-314.

Kho77 A. G. Khovanskii, Newton polyhedra and toroidal varieties, Funct. Anal. Appl. 11 (1977), 56-64. (English transl. Funct. Anal. Appl. 11 (1977), 289-296.)

KKMS73 G. Kempf, F. Knudsen, D. Mumford and B. St Donat, Toroidal embeddings, Lecture Notes in Mathematics, vol. 339 (Springer, Berlin, 1973).

KL77 T. C. Kuo and Y. C. Lu, On analytic function germs of two complex variables, Topology 16 (1977), 299-310.

KP04 T. C. Kuo and A. Parusinski, Newton-Puiseux roots of Jacobian determinants, J. Algebraic Geom. 13 (2004), 579-601.

Lip65 J. Lipman, Quasi-ordinary singularities of embedded surfaces, Thesis, Harvard University (1965).

Lip83 J. Lipman, Quasi-ordinary singularities of surfaces in $\mathbf{C}^{3}$, Proc. Sympos. Pure Math., vol. 40 (American Mathematical Society, Providence, RI, 1983), 161-172.

Lip88 J. Lipman, Topological invariants of quasi-ordinary singularities, Mem. Amer. Math. Soc. 74 (1988), 1-107.

LMW89 Lê D. T., F. Michel and C. Weber, Sur le comportement des polaires associées aux germes de courbes planes, Compositio Math. 72 (1989), 87-113. 


\section{DECOMPOSITION IN BUNCHES OF THE CRITICAL LOCUS OF A QUASI-ORDINARY MAP}

Mer77 M. Merle, Invariants polaires des courbes planes, Invent. Math. 41 (1977), 103-111.

MN03 L. J. McEwan, and A. Némethi, Some conjectures about quasi-ordinary singularities, Contemp. Math., vol. 324 (American Mathematical Society, Providence, RI, 2003), 185-193.

MN04 L. J. McEwan and A. Némethi, The zeta function of a quasi-ordinary singularity, Compositio Math. 140 (2004), 667-682.

Mum88 D. Mumford, The red book on varieties and schemes, Lecture Notes in Mathematics, vol. 1358, (Springer, Berlin, 1988).

Oda88 T. Oda, Convex Bodies and Algebraic Geometry, Ergeb. Math. Grenzgeb. (3), vol. 15 (Springer, Berlin, 1988).

Pop01 P. Popescu-Pampu, Arbres de contact des singularités quasi-ordinaires et graphes d'adjacence pour les 3-variétés réelles, Thèse, Université de Paris 7 (2001).

Pop04 P. Popescu-Pampu, Sur le contact d'une hypersurface quasi-ordinaire avec ses hypersurfaces polaires. J. Inst. Math. Jussieu 3 (2004), 105-138.

Ris03 J.-J. Risler, On the curvature of the real Milnor fiber, Bull. London Math. Soc. 35 (2003), 445-454.

Tei77a B. Teissier, The hunting of invariants in the geometry of discriminants, in Real and complex singularities (Proc. Ninth Nordic Summer School/NAVF Sympos. Math., Oslo, 1976) (Sijthoff and Noordhoff, Alphen aan den Rijn, 1977), 565-678.

Tei77b B. Teissier, Variétés polaires. I. Invariants polaires des singularités d'hypersurfaces, Invent. Math. 40 (1977), 267-292.

Tei80 B. Teissier, Polyèdre de Newton jacobien et équisingularité, in Seminar on Singularities (Paris, 1976/1977), Publ. Math. Univ. Paris VII 7 (Univ. Paris VII, Paris, 1980), 193-221.

Tei86 B. Teissier, The monomial curve and its deformations, Appendix in O. Zariski, Le problème des modules pour les branches planes (Hermann, Paris, 1986).

Vil00 O. Villamayor, On equiresolution and a question of Zariski, Acta Math. 185 (2000), 123-159.

Wal03 C. T. C. Wall, Chains on the Eggers tree and polar curves, Rev. Mat. Iberoamericana 19 (2003), $1-10$.

Zar55 O. Zariski, The connectedness theorem for birrational transformations, in Algebraic Geometry and Topology (Symposium in honor of S. Lefschetz) (Princeton University Press, Princeton, NJ, 1955), $182-188$.

Zar67 O. Zariski, Exceptional Singularities of an algebroid surface and their reduction, Atti Accad. Naz. Lincei Rend. Cl. Sci. Fis. Mat. Natur. (8) 43 (1967), 135-146; reprinted in Oscar Zariski collected papers, vol. 1 (MIT, Cambridge, MA, 1972).

E. R. García Barroso ergarcia@ull.es

Departamento de Matemática Fundamental, Facultad de Matemáticas, Universidad de La Laguna, 38271, La Laguna, Tenerife, Spain

\section{P. D. González Pérez pgonzalez@mat.ucm.es}

Departamento de Algebra, Facultad de Ciencias Matemáticas, Universidad Complutense de Madrid, Plaza de las Ciencias 3, 28040 Madrid, Spain 